\title{
Role of nonlocality and Landau damping in the dynamics of a quantum dot coupled to surface plasmons
}

\author{
A. Vagov,,${ }^{1, *}$ I. A. Larkin, ${ }^{2,3}$ M. D. Croitoru, ${ }^{4,5}$ and V. M. Axt ${ }^{1}$ \\ ${ }^{1}$ Theoretische Physik III, Universität Bayreuth, 95440 Bayreuth, Germany \\ ${ }^{2}$ Department of Physics, Minho University, 4710-057, Braga, Portugal \\ ${ }^{3}$ Institute of Microelectronics Technology Russian Academy of Sciences, 142432 Chernogolovka, Russia \\ ${ }^{4}$ Departement Fysica, Universiteit Antwerpen, B-2020 Antwerpen, Belgium \\ ${ }^{5}$ Universidade Federal de Pernambuco, 50670-901 Recife, Pernambuco, Brazil \\ (Received 25 January 2016; revised manuscript received 11 April 2016; published 10 May 2016)
}

\begin{abstract}
We investigate the dynamics of a quantum emitter (quantum dot) placed in the vicinity of a flat metal surface. The dynamics is induced by the coupling between the emitter and surface plasmon-polaritons. The plasmon-polariton modes are described within a continuous media model with a nonlocal Lindhard-type dielectric response of the metal. The analytic solution of the dynamical equations is obtained in the rotating wave approximation. The results demonstrate a considerable influence of the nonlocality of the electromagnetic response and the Landau damping in the metal. In particular, the relaxation dynamics is characterized by two distinct times that may differ by large amounts as a consequence of the nonlocality of the response. It is also shown that one of the contributions to the relaxation can have a power-law long-time asymptote, leading to notable changes in the dynamical pattern.
\end{abstract}

DOI: 10.1103/PhysRevB.93.195414

\section{INTRODUCTION}

Unique properties of surface plasmon-polariton (SPP) excitations [1-3] can be used in many applications, in particular to design sources of nonclassical light [4] and single photons [5-8], nanophotonic lasers [9-13], nonconventional optical metamaterials [14], nanoantennas [15,16] and efficient light sensors [17], transistors [18-20], and quantum information devices [21-23]. Many of those applications utilize the possibility of a precise manipulation of the electromagnetic fields associated with SPP modes. Unlike conventional light waves, SPPs resolve scales below the diffraction limit [24] which can be used to achieve a considerable enhancement of the field intensity and a better control of its spatial distribution [25]. The Achilles heel in some of the applications is the weak coupling between SPPs and external light. This can be remedied by introducing mediating scatterers (or emitters), such as, for example, J-aggregates [26,27], dye molecules [28], or strongly confined quantum dots [29], that are strongly coupled to both incoming light waves and surface plasmons. As a bonus, the strong coupling can be used to achieve coherent manipulation of the emitter quantum states.

Although SPP excitations involve a macroscopically large number of fermion carriers, they can to a good approximation behave as a quantum bosonic field [30-33] whose dynamics is induced by discrete creation and absorption of excitation quanta. The quantum nature of plasmons becomes important when the SPP-emitter interaction is strong and significant hybridization of SPP and emitter states takes place [34,35]. Achieving this regime of strong coupling is facilitated by the localization of SPP modes beyond the diffraction limit [24] as well as by a large local density of SPP states (DOS) via the Purcell effect [36]. The coupling strength and, respectively, the dynamics of the system can thus be controlled by geometrical parameters of the system [37]. Evidences of the strong

\footnotetext{
*alexei.vagov@uni-bayreuth.de
}

coupling regime have been recently observed by demonstrating anticrossings of spectral lines [38], as well as by revealing a boost in emission rates [39-41] and the vacuum Rabi splitting [42]. The recent observation of peculiar dynamical features in the ultrafast time scale is believed to be another evidence of the strong coupling [43].

The theoretical analysis of the dynamics of a quantum emitter coupled with SPPs can be split into two parts: (1) the description of SPPs and their coupling with the emitter and (2) the calculation of the coupled dynamics. It should be noted that such systems belong to a general class of quantum dissipative systems [44,45], where SPP modes act as a continuum of reservoir states. The dynamics of those systems strongly depends on properties of the continuum and is thus sensitive to the details of the model and to the approximations used in the analysis.

An $a b$ initio description of SPP modes requires solving Maxwell equations coupled to the microscopic equations for charge carriers in the media. Density functional theory has been recently applied to describe SPPs for an arbitrary geometry of the interface [46-48]. However, extending this approach to the dynamics involving the interaction with an external emitter appears to be too complicated. Most theoretical studies of SPP modes are done with simplifying assumptions.

A common approach is to use a continuous media model combined with the linear response theory. It neglects changes of the carrier states in the vicinity of the interface but simplifies the analysis by separating the material and the Maxwell equations. Finding electromagnetic fields in polarized media of arbitrary shape is still not an easy task. The solution is considerably simpler when the dielectric response is taken to be local and spatially independent within the respective media. This local approximation is used in most textbook analyses of SPPs [49] and can be, in principle, extended to any interface geometry. However, it introduces unwanted modifications in the dynamics via an artificial singularity in the SPP DOS. The nonlocality is important at distances comparable with the mean-free path of the metal carriers. However, the 
electromagnetic problem without the local approximation can be solved only for few simple geometries such as a flat surface [50,51], a slab [52], and, with some reservations, a sphere [53-56].

In the subsequent analysis of the dynamics, SPP modes, which are obtained from separate calculations, are used as an input. Many earlier works assumed that the time evolution is well described by an exponential decay and the calculations were restricted to obtain the decay rate [50-52,57-66]. This approximation neglects quantum coherence and non-Markovian memory effects, but is admissible when the coupling is weak, SPPs decay faster than the emitter states, and one is interested in the evolution at long times. Its validity domain can be estimated by using the Nakajima-Zwanzig expansion $[63,67]$ which indicates that the approach can be used at times $t \gtrsim 1 \mathrm{ps}$ for a typical metal. However, at shorter times quantum coherence and memory effects cannot be neglected.

Most of the recent research of the dynamics used simplified descriptions for SPPs such as a harmonic oscillator continuum model [57], a model with fixed dielectric constants [58,60,64], the hydrodynamic Ritchie model [61], the Drude model of the dielectric response $[59,62,63,65]$, etc. The relaxation processes are often taken into account by introducing phenomenological decay rates, which is equivalent to the Markov approximation.

We point to a general trend that a more accurate analysis of the dynamics is usually complemented by a less realistic modeling of SPPs and vice versa. In particular, the role of the Landau damping in the time evolution is often overlooked, although it yields the fastest relaxation rate when a quantum emitter is close to a metallic surface [51,52]. The known sensitivity of the dynamics of quantum dissipative systems on the structure of the continuum implies that neglecting this and other features of SPP modes gives rise to a qualitatively different dynamical pattern.

In this work, we present an accurate analysis of the dynamics of a quantum dot (QD) with few carrier states coupled to SPPs described within the Lindhard-Mermin model of the dielectric response [68-71]. The calculations account for both the nonlocality and the Landau damping, which give rise to novel dynamical features, that cannot be described by rate models. In particular, the dynamics is characterized by two different relaxation mechanisms and can have a power-law long-time asymptote. The calculations are done using material parameters of a prototype InGaAs dot embedded in a GaAs insulator and placed in front of a half-space filled with silver. A discussion of the validity and limitations of the used approach is given in the Appendix.

\section{THEORETICAL MODEL}

We consider a QD, embedded in an insulating material and placed at a distance $a$ from a flat metallic surface, as schematically illustrated in Fig. 1. The QD is assumed to be strongly confined, so that the energy levels of its carrier states are well separated. For simplicity, the analysis will be done for the case of two optically active states, the ground state $|G\rangle$ and an excited state $|E\rangle$. The QD is initially in the excited state (for example, excited by applying an ultrashort laser pulse) and its subsequent time evolution is induced by its coupling with the charge carriers in the metal via the electromagnetic field. The strongest coupling is achieved between the QD and SPP

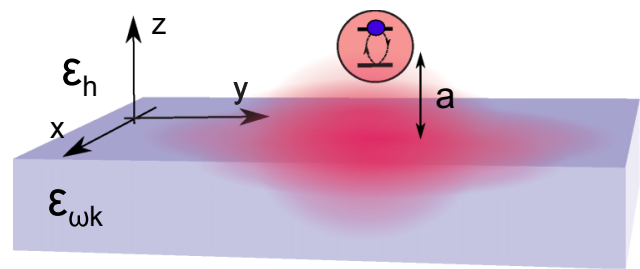

FIG. 1. A schematic illustration of the system: a quantum dot with two optically active states, embedded in a material with dielectric constant $\varepsilon_{h}$, is placed at the distance $a$ from the surface of a metal with the permittivity function $\varepsilon_{\omega k}$. Surface plasmons, excited by electromagnetic coupling with the metal, propagate in the $x-y$ plane.

modes, that are localized in the vicinity of the metal-insulator interface.

We construct an effective quantum Hamiltonian that takes into account SPP excitations, QD states, and their interaction. The SPP Hamiltonian is obtained by using a standard representation of a quantized bosonic field [72,73] of collective plasma excitations proposed in early works of Pines [30], Hopfield [31], and Ritchie [32,33]. SPP eigenmodes are found by solving Maxwell's equations for a continuous media model in the geometry shown in Fig. 1. The solution is used to calculate the SPP frequency dispersion and the QD-SPP coupling constants.

\section{A. Solution of Maxwell equations}

SPP modes are found by solving Maxwell equations for the field combined with the material equations for the media charge carriers. We assume a linear electromagnetic response of the media so that the polarization $\mathbf{P}$ in both materials is given as a functional of the form

$$
\mathbf{P}(\mathbf{r}, t)=\int \check{\chi}\left(\mathbf{r}, \mathbf{r}^{\prime}, t-t^{\prime}\right) \mathbf{E}\left(\mathbf{r}^{\prime}, t^{\prime}\right) d \mathbf{r}^{\prime} d t^{\prime} .
$$

One can safely assume that the dielectric response of the insulator is local and can be approximated by a single constant for an isotropic material, so that for $z>0 \mathrm{Eq}$. (1) reduces to

$$
\mathbf{P}=\chi_{h} \mathbf{E} .
$$

In contrast, the nonlocality of the response of the metal cannot be neglected when the characteristic dimensions in the system are comparable with the mean-free path of its delocalized charge carriers.

For bulk systems, the nonlocality of the response arises because the electrical susceptibility tensor $\chi_{B}$ which describes the reaction to a plane-wave excitation propagating with wave vector $\mathbf{k}$ and frequency $\omega$ depends on $\mathbf{k}$ and $\omega$. This translates to a nonlocal susceptibility $\chi_{B}\left(\mathbf{r}-\mathbf{r}^{\prime}, t-t^{\prime}\right)$ in space and time which is connected with $\chi_{B_{\omega \mathbf{k}}}$ by a Fourier transformation. For a simple isotropic metal $\chi_{B}$ is proportional to the unit tensor.

Treating the metal electrons in mean-field approximation and using a single-band model the linear response can be obtained by solving the corresponding quantum mechanical problem leading to the well-known Lindhard formula [70,74]. Assuming a parabolic band structure with effective mass $m$ and concentrating on wave vectors $\mathbf{k}$ with $|\mathbf{k}|$ much smaller than the Fermi wave number $k_{F}$, which is in our calculations 
always satisfied, the Lindhard electric susceptibility simplifies to

$$
\chi_{\omega \mathbf{k}}=\frac{3 \Omega_{p}^{2}}{k^{2} v_{F}^{2}}\left(1-\frac{1}{2} \frac{\omega}{k v_{F}} \ln \left[\frac{\omega+k v_{F}}{\omega-k v_{F}}\right]\right),
$$

where $v_{F}$ is the Fermi velocity and $\Omega_{p}=\sqrt{n e^{2} / m}$ is the bulk plasma frequency. Unlike the Drude model, which can be obtained from Eq. (3) in the limit $k \rightarrow 0$, this expression retains the $k$ dependence and thus exhibits a genuine nonlocality in real space.

Note that the imaginary part of Eq. (3) becomes nonzero for $k>\omega / v_{F}$. This is a manifestation of the Landau damping, which describes the decay of plasmons that travel at the Fermi velocity via excitation of electron-hole pairs [49,71,74]. Other relaxation mechanisms, e.g., due to the finite lifetime $\tau_{e}=1 / \Gamma$ of quasiparticles in the metal, can be accounted phenomenologically by amending the quantum kinetic equation used to derive Eq. (3). This must be done accounting for the particle conservation law, which yields the corrected Lindhard-Mermin expression [69]

$$
\tilde{\chi}_{\omega \mathbf{k}}=\frac{(1+\eta)\left(1+\chi_{\bar{\omega} \mathbf{k}}\right)}{1+\eta \chi_{\bar{\omega} \mathbf{k}} / \chi_{0 \mathbf{k}}},
$$

where $\eta=\AA \mathbb{1}\left(\omega \tau_{e}\right)^{-1}$ and $\bar{\omega}=(1+\eta) \omega$. In the limit $k \rightarrow 0$, Eq. (4) gives the Drude susceptibility

$$
\tilde{\chi}_{\omega}=-\frac{\Omega_{p}^{2}}{\omega^{2}(1+\eta)},
$$

with the correct (phenomenological) account for the relaxation [68]. In what follows, we assume that in the interval of interest $\eta \ll 1$.

The presence of a surface breaks the translational symmetry which in a material with a nonlocal response should lead to modifications of the response functions. To see this more clearly, imagine a fictitious electric point source placed at a point $\mathbf{r}_{0}$ inside such a material. In bulk geometry, this source will generate a polarization $\mathbf{P}(\mathbf{r})$ that according to Eq. (1) spreads isotropically away from the source with an amplitude $\sim \chi_{B}\left(\mathbf{r}-\mathbf{r}_{0}\right)$. However, the surface will interrupt this spreading. A simple way to account phenomenologically for the influence of the interface on the susceptibility is provided by the so-called specular reflection model (SRM), which assumes that the main modification is captured by a mirror reflection of the outgoing polarization wave at the surface [51,53]. Technically, this means that the polarization current arising as a response to an electric field in the metal is composed of two components traveling to and from the surface (reflected current) [75]. The phase factor of the reflected component must be chosen so that the normal current at the interface is zero to ensure that no carriers leave the metal. The tangential current is assumed to be unrestricted leading to a reflection without phase jump. With these assumptions, $\check{\chi}$ for the half-space metallic sample is found as

$$
\check{\chi}=\left(\begin{array}{ccc}
\chi_{+} & 0 & 0 \\
0 & \chi_{+} & 0 \\
0 & 0 & \chi_{-}
\end{array}\right)
$$

where the components of this tensor are given as combinations of the bulk susceptibility $\chi_{B}$ of the same material

$$
\chi_{ \pm}=\tilde{\chi}\left(\rho-\rho^{\prime}, z-z^{\prime}\right) \pm \tilde{\chi}\left(\rho-\rho^{\prime}, z+z^{\prime}\right),
$$

where we introduced a notation $\rho=(x, y)$. Finally, in a halfspace model the $\mathbf{r}^{\prime}$ integration in Eq. (1) over the sources of the polarization needs to be restricted to the half-space $z^{\prime}<0$.

The SRM has been previously employed to describe reflections of electromagnetic waves from interfaces between different materials as well as to describe SPPs in the quasistatic limit $[51,52,54,55]$. Within the SRM the material equations satisfy additional symmetry which can be utilized by introducing auxiliary boundary conditions (ABC approach) which greatly simplify the solution [53,76]. In fact, substituting the representation in Eq. (1), one can see that the electrodynamic problem in the half-space can be formally extended to the full space with the fields at $z>0$ and at $z<0$ being mirrorreflected. The solution of Maxwell's equations is then obtained as in the bulk with a self-consistently determined surface charge arising from the jump of the polarization at $z=0$. In this way, explicit analytical solutions for the SPP modes can be obtained. While details of the derivation will be published elsewhere, here we shall only briefly summarize the main results.

There are two types of solution localized in the vicinity of the surface: (1) with the in-plane magnetic field perpendicular to the surface plasmon wave vector $\mathbf{q}=\left(k_{x}, k_{y}, 0\right)$ ( TM mode) and (2) with the in-plane electric field perpendicular to $\mathbf{q}$ (TE mode). It can be shown that the TE mode is overdamped (its eigenfrequency is imaginary). For the TM solution with frequency $\omega$ and wave vector $\mathbf{q}$ (without loss of generality, we assume $\mathbf{q} \| \mathbf{e}_{x}$ ), the fields inside the metal read as

$$
\begin{aligned}
& B_{y}=-e^{\mathfrak{i} q x} \frac{\sigma_{\omega \mathbf{q}} \bar{\omega}}{q} \int_{-\infty}^{\infty} e^{\mathfrak{i} k_{z} z} k_{z} G_{\omega \mathbf{k}} d k_{z}, \\
& E_{x}=e^{\mathfrak{i} q x} \frac{\sigma_{\omega \mathbf{q}}}{q} \int_{-\infty}^{\infty} e^{\mathfrak{i} k_{z} z} \frac{q^{2}-\varepsilon_{\omega \mathbf{k}} \bar{\omega}^{2}}{\varepsilon_{\omega \mathbf{k}}} G_{\omega \mathbf{k}} d k_{z}, \\
& E_{z}=e^{\mathfrak{i} q x} \sigma_{\omega \mathbf{q}} \int_{-\infty}^{\infty} e^{\mathfrak{i} k_{z} z} \frac{k_{z}}{\varepsilon_{\omega \mathbf{k}}} G_{\omega \mathbf{k}} d k_{z},
\end{aligned}
$$

where $\bar{\omega}=\omega / \mathrm{c}, \sigma_{\omega \mathbf{q}}$ is the mode amplitude, and

$$
G_{\omega \mathbf{k}}=-\frac{4 \pi \stackrel{\circ}{1}}{k^{2}-\varepsilon_{\omega k} \bar{\omega}^{2}} .
$$

In the above equations, $\varepsilon_{\omega \mathbf{k}}=1+\tilde{\chi}_{\omega \mathbf{k}}$ is the dielectric permittivity of the bulk (homogeneous and isotropic) metal.

The fields at $z>0$ inside the insulator are obtained similarly and are given by Eqs. (8) with the substitutions $\varepsilon_{\omega k} \rightarrow \varepsilon_{h}$ and $\sigma_{\omega \mathbf{q}} \rightarrow \sigma_{\omega \mathbf{q}}^{\prime}$. The total solution is found by matching the boundary conditions at the interface $z=0$. The conditions for $B_{y}$ and $E_{z}$ yield $\sigma_{\omega \mathbf{q}}^{\prime}=-\sigma_{\omega \mathbf{q}}$, while the condition for the $E_{x}$ component determines the frequency dispersion of the SPP modes discussed in the next subsection. 


\section{B. SPP frequency dispersion}

The appearance of the Lindhard expression makes it possible to introduce dimensionless quantities

$$
\tilde{\omega}=\frac{\omega}{\Omega_{p}}, \quad \tilde{t}=\Omega_{p} t, \quad \tilde{\mathbf{k}}=\frac{v_{F}}{\Omega_{p}} \mathbf{k}, \quad \tilde{\mathbf{r}}=\frac{\Omega_{p}}{v_{F}} \mathbf{r},
$$

that we shall use in the following calculations, omitting the "tilde" unless this causes confusion. Equating $E_{x}$ given by Eq. (8) on both sides of the interface at $z=0$ one obtains the dispersion equation

$$
\int_{-\infty}^{\infty} \frac{1}{\varepsilon_{\omega k}} \frac{q^{2}-\varepsilon_{\omega k} \alpha^{2} \omega^{2}}{k^{2}-\varepsilon_{\omega k} \alpha^{2} \omega^{2}} d k_{z}=-\frac{\pi}{\varepsilon_{h}} \sqrt{q^{2}-\varepsilon_{h} \alpha^{2} \omega^{2}},
$$

where $\alpha=v_{F} / \mathrm{c}$.

Solutions of Eq. (11) can be obtained only numerically. However, concentrating on small $q$ values it is instructive to perform an expansion with respect to the two small parameters $\alpha \ll 1$ and $q \ll 1$. The leading-order contribution to Eq. (11) gives rise to the standard dispersion for SPP modes in the Drude limit, where

$$
\varepsilon_{\omega} \equiv \varepsilon_{\omega k \rightarrow 0}=1-\frac{1}{\omega^{2}(1+\eta)} .
$$

We denote the corresponding solution for the dispersion as $\omega_{q}^{(0)}$. Corrections to $\omega_{q}^{(0)}$ can be obtained by subtracting the identity

$$
\int_{-\infty}^{\infty} \frac{q^{2}-\varepsilon_{\omega} \alpha^{2} \omega^{2}}{k^{2}-\varepsilon_{\omega} \alpha^{2} \omega^{2}} \frac{d k_{z}}{\varepsilon_{\omega}}=\frac{\pi}{\varepsilon_{\omega}} \sqrt{q^{2}-\varepsilon_{\omega} \alpha^{2} \omega^{2}},
$$

from both sides of Eq. (11). Expanding the result with respect to $\alpha$ and keeping the two largest contributions, we obtain the equation

$$
\frac{q^{2}}{\pi} \int_{-\infty}^{\infty}\left(\frac{1}{\varepsilon_{\omega k}}-\frac{1}{\varepsilon_{\omega}}\right) \frac{d k_{z}}{k_{z}^{2}+q^{2}}=-\frac{q}{\varepsilon_{\omega}}-\frac{q}{\varepsilon_{h}}+\frac{\alpha^{2} \omega^{2}}{q} .
$$

Expanding this further with respect to $q \ll 1, \eta \ll 1$ and taking the largest terms, one arrives at the dispersion equation in a simplified form

$$
q \beta(\omega)+\frac{1}{\varepsilon_{\omega}}+\frac{1}{\varepsilon_{h}}=\frac{\alpha^{2} \omega^{2}}{q^{2}},
$$

where

$$
\beta(\omega)=\frac{1}{\pi} \lim _{q \rightarrow 0} \lim _{\eta \rightarrow 0}\left[\int_{-\infty}^{\infty}\left(\frac{1}{\varepsilon_{\omega k}}-\frac{1}{\varepsilon_{\omega}}\right) \frac{d k_{z}}{k^{2}}\right] .
$$

One notes that with $\beta(\omega)=0$, Eq. (15) recovers the dispersion equation in the Drude model [49]. Its solution $\omega_{q}^{(0)}$ saturates at large $q$ reaching the constant surface plasmon frequency $\Omega_{s p}=1 / \sqrt{1+\varepsilon_{h}}$. In the opposite limit of small $q$ one has $\omega_{q}^{(0)} \simeq q /\left(\alpha \sqrt{\varepsilon_{h}}\right)$ which coincides with the dispersion of light propagating in the insulator with the dielectric constant $\varepsilon_{h}$. These two limiting cases interchange around $q_{0} \sim \alpha \sqrt{\varepsilon_{h} /\left(1+\varepsilon_{h}\right)} \ll 1$.

Equation (15) can be solved perturbatively by taking into account that $\beta(\omega)$ is small. Seeking the solution in the form

$$
\omega_{q}=\omega_{q}^{(0)}\left(1+\Delta_{q}\right)
$$

we substitute it into Eq. (15) and then expand the result with respect to $\beta(\omega)$, which yields the correction

$$
\Delta_{q} \approx \frac{\varepsilon_{h}^{2} \beta\left(\omega_{q}^{(0)}\right)}{1+\varepsilon_{h}} \frac{q}{2}-\frac{\AA}{2} \frac{\Gamma}{\omega_{q}^{(0)}} .
$$

This can be further simplified at $q \gtrsim q_{0}$, where one can use the quasistatic approximation $\omega_{q}^{(0)} \simeq \Omega_{s p}$ [51] (the validity of this approximation is discussed in Appendix). Thus, at $q \gtrsim q_{0}$, Eqs. (17) and (18) give a simple linear dispersion

$$
\omega_{q} \approx \Omega_{s p}-\frac{\AA}{2} \Gamma+v_{p} q,
$$

where the velocity $v_{p}$ is given by Eq. (18) with $\omega_{q}^{(0)} \rightarrow \Omega_{s p}$. The integral in Eq. (16) is estimated by separating real and imaginary parts in the integrand and then expanding its imaginary part with respect to large $k / \omega \gg 1$. This yields

$$
v_{p}=\frac{\varepsilon_{h}^{2}}{\left(1+\varepsilon_{h}\right)^{2}}\left(1-\frac{\AA}{12} \frac{\ln \left[1+\varepsilon_{h}\right]}{\sqrt{1+\varepsilon_{h}}}\right) .
$$

One notes that the obtained dispersion (19) is complex, $\omega_{q}=\omega_{q}^{\prime}+i \omega_{q}^{\prime \prime}$, even when one neglects other relaxation processes, $\Gamma=0$, because of a nonzero imaginary part of

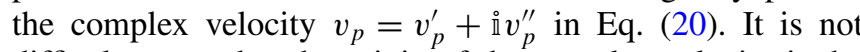
difficult to see that the origin of the complex velocity is the Landau damping, manifested in the logarithmic singularity in the Lindhard expression. The corresponding damping rate rises linearly with the plasmon wave vector, so that the contribution of the Landau damping increases, becoming the largest decay channel at larger $q$. However, direct calculations reveal that the SPP modes are still weakly damped, $\omega_{q}^{\prime \prime} \ll \omega_{q}^{\prime}$, in the entire validity domain of Eq. (19).

We also stress that the linear character of the SPP dispersion at larger $q$ is a consequence of the nonlocality of the dielectric function, which is manifested in a finite value of $\beta$. Moreover, the SPP group velocity at $q \gtrsim q_{0}$ can be estimated as $v_{p}^{\prime} \sim 1$ ( $v_{p}^{\prime} \sim v_{F}$ in the original units), which is considerably smaller than the velocity of the polariton propagation $\mathrm{c} / \sqrt{\varepsilon_{h}}$ at $q \lesssim q_{0}$. An important consequence of this fact is that the SPPs DOS at $\omega \sim \Omega_{s p}$ is much larger than at $\omega \ll \Omega_{s p}$. The relation between the linear plasmon dispersion at $q \gtrsim q_{0}$ and the nonlocality of the dielectric response was noted earlier by calculating the plasmon dispersion in the quasistatic approximation for the Maxwell equations, which gives numerical results close to ours for a metal-vacuum interface $\left(\varepsilon_{h}=1\right)$ [76].

Figure 2 compares results for the real part of the dispersion obtained by solving Eq. (11) numerically and the linear dependence in Eqs. (19) (the calculations are done for the silver material parameters discussed later in Sec. IV). The dispersion $\omega_{q}^{(0)}$ is also shown for comparison. The results demonstrate a good accuracy of the linear approximation at $q \gtrsim q_{0}$.

\section{Quantized SPP modes and QD coupling}

Weakly damped SPP excitations in fact represent an ensemble of independent harmonic oscillators. Such excitations can be quantized using bosonic field operators $\hat{a}_{\mathbf{q}}$ and $\hat{a}_{\mathbf{q}}^{\dagger}$, which act in the usual way in the space of the SPP mode occupation numbers [30-33]. The quantum Hamiltonian of 


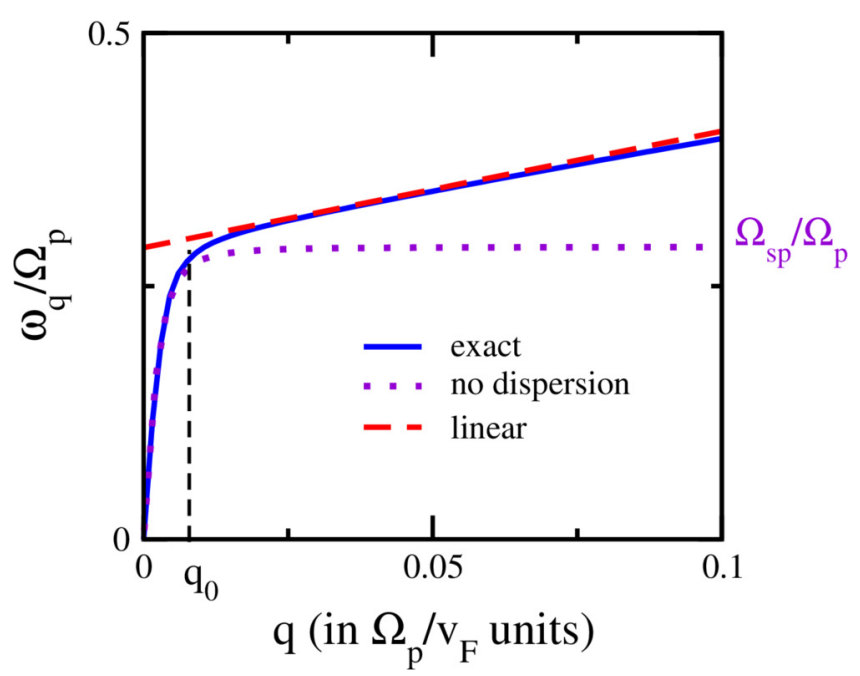

FIG. 2. Real part of the SPP frequency dispersion $\omega_{q}^{\prime}$. Continuous (blue) line, labeled "exact," gives the numerical solution of Eq. (11). Dotted (violet) line, labeled "no dispersion," is the result obtained for the local approximation for the permittivity $\varepsilon_{\omega}$, which yields the limiting value of the plasmon frequency $\Omega_{s p} / \Omega_{p}=1 / \sqrt{1+\varepsilon_{h}} \approx$ $0.29\left(\varepsilon_{h}=11\right)$. Dashed (red) line, labeled "linear," shows the linear dispersion in Eq. (19). All quantities are shown in scaled units defined in Eq. (10), the material parameters are typical for silver and discussed in the text.

the SPP subsystem writes as

$$
\hat{H}_{\mathrm{SPP}}=\sum_{\mathbf{q}} \hbar \omega_{\mathbf{q}}^{\prime}\left(\hat{a}_{\mathbf{q}}^{\dagger} \hat{a}_{\mathbf{q}}+\frac{1}{2}\right) .
$$

Here, we took into account only the real part $\omega_{\mathbf{q}}^{\prime}$ of the SPP frequency, while its imaginary part $\omega_{q}^{\prime \prime}$, which describes the damping of SPP oscillations, can be taken into account, e.g., by introducing an interaction between SPP modes and other system excitations. However, when the damping is weak, $\omega_{q}^{\prime \prime} \ll \omega_{\mathbf{q}}^{\prime}$, its influence on the dynamics can be studied phenomenologically by appropriate modifications of the dynamical equations.

The quantum interaction Hamiltonian between SPP modes and the QD states assumes the form [49,72,73]

$$
\hat{V}=-\hat{\mathbf{E}} \cdot \hat{\mathbf{P}},
$$

where $\hat{\mathbf{P}}$ is the operator of the QD dipole moment and $\hat{\mathbf{E}}$ is the electric field operator. We assume that the contribution of the SPP modes to the electric field operator can be written in terms of the creation/destruction operators $\hat{a}_{\mathbf{q}}$ and $\hat{a}_{\mathbf{q}}^{\dagger}$ for the SPP modes as

$$
\hat{\mathbf{E}}=\sum_{\mathbf{q}}\left(\mathbf{E}_{\mathbf{q}} \hat{a}_{\mathbf{q}}+\mathbf{E}_{\mathbf{q}}^{*} \hat{a}_{\mathbf{q}}^{\dagger}\right),
$$

where the field $\mathbf{E}_{\mathbf{q}}$ associated with the excitation of the SPP mode $\mathbf{q}$ (now the direction of $\mathbf{q}$ is arbitrary) is given by Eq. (8). The resulting expression for the QD-SPP interaction contains the amplitudes $\sigma_{\omega \mathbf{q}}$ which still need to be fixed. To this end, we note that the energy of weakly damped SPP modes can be well approximated by the energy of the corresponding electromagnetic field in the media. Other contributions due to, e.g., changes in the particles kinetic energy, can be neglected in this case. For the electromagnetic energy of a single (nonquantized) SPP mode, we use the following standard expression for the field energy in dispersive linear responding media [73]:

$$
W_{q}=\frac{1}{16 \pi} \int\left\{\mathbf{E}_{q}^{*} \frac{\partial(\omega \hat{\varepsilon})}{\partial \omega} \mathbf{E}_{q}+\mathbf{B}_{q}^{*} \mathbf{B}_{q}\right\} d r,
$$

where the integrals are calculated separately in the half-spaces $z \lessgtr 0$ for the metal and for the insulator. We interpret $W_{q}$ as the energy of a single excitation of the SPP mode $q$ which allows us to determine $\sigma_{\omega \mathbf{q}}$ by demanding for consistency that $\hbar \omega_{q}^{\prime}=W_{q}$.

Substituting the solution of the TM mode from Eq. (8), we obtain the energy as the sum of four components:

$$
W_{q}=W_{q, E}^{(+)}+W_{q, E}^{(-)}+W_{q, B}^{(+)}+W_{q, B}^{(-)},
$$

where " \pm " denotes contributions by the insulator $(z>0)$ and the metal $(z<0)$, given as

$$
\begin{aligned}
W_{q, E}^{( \pm)}= & \frac{S}{32 \pi} \int_{-\infty}^{\infty} \frac{d k_{z}}{2 \pi} \operatorname{Re}\left[\frac{\partial\left(\omega \varepsilon_{ \pm}\right)}{\partial \omega}\right] \\
& \times\left\{\left|E_{x}^{( \pm)}\right|^{2}+\left|E_{z}^{( \pm)}\right|^{2}\right\}, \\
W_{q, B}^{( \pm)}= & \frac{S}{32 \pi} \int_{-\infty}^{\infty}\left|B_{y}^{( \pm)}\right|^{2} \frac{d k_{z}}{2 \pi},
\end{aligned}
$$

where $\varepsilon_{+}=\varepsilon_{h}, \varepsilon_{-}=\varepsilon_{\omega k}$, and $S$ is the area of the surface (set to infinity in the end).

Further, we neglect a small interval $k_{z} \lesssim q_{0} \sim \alpha \ll 1$ in the integral in Eq. (26), where the spectrum is of the polariton (lightlike) type and the SPP DOS is small. We also note that in the interval $q \gtrsim q_{0}$, the magnetic field contribution contains a small factor $\propto \alpha \omega / q \ll 1$ and can be neglected. The electric field contribution is calculated with the same accuracy as assumed for the SPP dispersion. This yields

$$
W_{q, E}^{(-)}=\frac{S \sigma_{\omega \mathbf{q}}^{2}}{4} \operatorname{Re}\left[\left(1-\omega \frac{\partial}{\partial \omega}\right) \int_{-\infty}^{\infty} \frac{1}{\varepsilon_{\omega k}} \frac{d k_{z}}{k_{z}^{2}+q^{2}}\right],
$$

where we set $\omega=\omega_{q}$ in the end of the calculations. Then, we expand the integrand with respect to small $q$ and retain the two leading contributions. The expansion is conveniently done after adding to and subtracting from Eq. (27) the same expression with the substitution $\varepsilon_{\omega k} \rightarrow \varepsilon_{\omega}$. The result reads as

$$
W_{q, E}^{(-)}=\frac{S \sigma_{\omega \mathbf{q}}^{2}}{4 q} \operatorname{Re}\left[\left(1-\omega \frac{\partial}{\partial \omega}\right)\left(\frac{1}{\varepsilon_{\omega}}-q \beta(\omega)\right)\right],
$$

where $\beta(\omega)$ is given by Eq. (16). The contribution by the insulator $W_{q, E}^{(+)}$is obtained similarly from Eq. (27) by substituting $\varepsilon_{\omega} \rightarrow \varepsilon_{h}$ and by setting $\beta(\omega)=0$.

Using Eqs. (15), (19), and (20) to simplify Eq. (28) we obtain from the condition $\hbar \omega_{q}^{\prime}=W_{q}$ the mode amplitude as

$$
\sigma_{\omega \mathbf{q}}=\frac{\hbar \omega_{q}}{Z_{q}}
$$

where the normalization factor $Z_{q}$, calculated to terms linear in $q$, is given by

$$
Z_{q}=\frac{\pi S\left(1+\varepsilon_{h}\right)}{2 \varepsilon_{h}^{2} q}\left\{1+\frac{2 \varepsilon_{h}\left(2+\varepsilon_{h}\right) q}{\left(1+\varepsilon_{h}\right)^{3 / 2}}\right\} .
$$


Finally, using this normalization in Eq. (8) the electric field associated with the SPP mode in the insulator can be written as

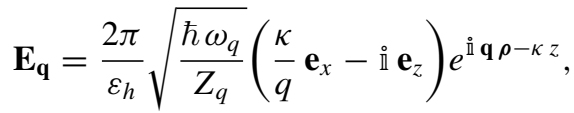

where $\kappa^{2}=q^{2}-\varepsilon_{h} \alpha^{2} \omega_{q}^{2}$.

We can now calculate the matrix elements of $\hat{V}$ between the quantum states, constructed as products of SPP and QD states. For the SPP subsystem the quantum states are characterized by the mode occupation numbers. We shall need the matrix elements between SPP states $n_{\mathbf{q}}$ and $n_{\mathbf{q}} \pm 1$ and the QD has states $|G\rangle$ and $|E\rangle$. The matrix elements can be formally written as $[77,78]$

$$
\begin{aligned}
\gamma_{\mathbf{q}} & =\left\langle n_{\mathbf{q}}, \Psi_{a}|\hat{V}| n_{\mathbf{q}}+1, \Psi_{b}\right\rangle \\
& =\int \Psi_{a}^{*}(\mathbf{r}) \Psi_{b}(\mathbf{r})\left(\mathbf{P} \cdot \mathbf{E}_{\mathbf{q}}(\mathbf{r})\right) d \mathbf{r},
\end{aligned}
$$

where $\mathbf{P}=e\left\langle\chi_{e}|\mathbf{r}| \chi_{h}\right\rangle$ is the dipole matrix element calculated between the microscopic Bloch electron (hole) states $\left|\chi_{e}(h)\right\rangle$, $e$ is the electron charge, and $\Psi_{a(b)}$ are the envelope wave functions of the QD states.

The microscopic calculation of $\mathbf{P}$ is beyond the scope of this work and is actually not needed. Its absolute value $p$ can be estimated from the available experimental data for the lifetime of QD excitons. The direction of $\mathbf{P}$ depends on the QD growth direction, the crystal symmetry, and the involved bands. It is, e.g., $\mathbf{P}=p / \sqrt{2}(1,0, \pm$ i $)$ for a $\sigma_{ \pm}$transition from heavy-hole states in GaAs-type QDs, when the growth direction is along the $y$ axis.

The integral in Eq. (32) is easily estimated when the QD size $d$ is much smaller than its distance $a$ to the surface $d \ll a$. Then, the product $\Psi_{a}^{*}(\mathbf{r}) \Psi_{b}(\mathbf{r})$ can be approximated by the Dirac delta function. Combining Eqs. (31), (30), (32), and (19), expanding the result with respect to small $q$, and retaining, as before, the two leading orders in the expansion, we obtain the square of the coupling constant as

$$
\left|\gamma_{\mathbf{q}}\right|^{2}=\frac{8 \pi}{S} \frac{p^{2} q e^{-2 q a}}{\left(1+\varepsilon_{h}\right)^{3 / 2}}\left\{1-\frac{\varepsilon_{h}\left(4+\varepsilon_{h}\right) q}{\left(1+\varepsilon_{h}\right)^{3 / 2}}\right\} .
$$

As mentioned, $p$ is estimated from the experimentally measured QD exciton lifetime $\tau_{0}=1 / \gamma_{0}$ using the standard relation between the dipole moment and radiative decay of the pointlike dipole [73]

$$
\gamma_{0}=\frac{4}{3} \frac{p^{2}}{\lambda_{e x}^{3}}
$$

where the wavelength $\lambda_{e x}=\mathrm{c} / \Omega_{e x}$ corresponds to the dot transition frequency $\Omega_{e x}$.

\section{Effective Hamiltonian}

Using the results of the previous subsection one can write a simple effective quantum Hamiltonian, which describes QD states, SPP bosonic excitations, and their coupling as

$$
\hat{H}=\hat{H}_{\mathrm{QD}}+\hat{H}_{\mathrm{SPP}}+\hat{H}_{\mathrm{QD}-\mathrm{SPP}},
$$

where the Hamiltonian of SPP modes has been defined earlier in Eq. (21), the Hamiltonian of the quantum dot is given by

$$
\hat{H}_{\mathrm{QD}}=\frac{\hbar \Omega_{e x}}{2}\left(1+\sigma_{z}\right),
$$

where $\sigma_{i}$ denote Pauli matrices acting in the space of the QD states, $\hbar \Omega_{e x}$ is the QD transition energy, and the interaction between QD and SPP modes is described by

$$
\hat{H}_{\mathrm{QD}-\mathrm{SPP}}=\sigma_{x} \sum_{\mathbf{q}} \hbar\left(\gamma_{\mathbf{q}} \hat{a}_{\mathbf{q}}+\gamma_{\mathbf{q}}^{*} \hat{a}_{\mathbf{q}}^{\dagger}\right),
$$

with the coupling constants $\gamma_{\mathbf{q}}$ defined in the previous section. The Hamiltonian in Eq. (35) neglects the interaction of the QD with an external laser field. Instead, we assume that a laser pulse prepares the QD in the excited state quasiinstantaneously, and then the QD evolves with time due to the interaction with SPP states.

In this model, we neglected pure dephasing processes that describe scatterings, e.g., with acoustic phonons, without changing a QD state. Such interactions lead to dephasing typically on a time scale of a few picoseconds while the dynamics to be discussed in the following takes place on a subpicosecond time scale. Furthermore, our Hamiltonian neglects the decay of SPP modes which will be taken into account phenomenologically in the analysis of the dynamics.

We finally stress that the model Hamiltonian comprises only TM modes, while the overdamped TE modes are neglected. Although strongly damped excitations can in principle create an effective relaxation channel, in practice the coupling to such excitations is weak.

\section{TIME EVOLUTION}

The system dynamics is studied by calculating the time evolution of the QD that is initially in its excited state $|E\rangle$. The solution to this problem can be easily found analytically in the rotating wave approximation (RWA) which neglects the transitions, where the creation/destruction of an SPP excitation is accompanied by the creation/destruction of the QD exciton [72]. The RWA approximation modifies the interaction part of the Hamiltonian to

$$
\hat{H}_{\mathrm{QD}-\mathrm{SPP}}^{\mathrm{RWA}}=\sum_{\mathbf{q}} \hbar\left\{\gamma_{\mathbf{q}} \sigma_{+} \hat{a}_{\mathbf{q}}+\gamma_{\mathbf{q}}^{*} \sigma_{-} \hat{a}_{\mathbf{q}}^{\dagger}\right\},
$$

with $\sigma_{ \pm}=\left(\sigma_{x} \pm \Uparrow \sigma_{y}\right) / 2$. It is assumed that before the dynamics started $(t<0)$, the SPP subsystem is in its ground state $\left(n_{q}=0\right)$. This assumption is justified when the temperature of the system is small in comparison with the average energy of SPP states, $T \ll \hbar\left\langle\omega_{q}\right\rangle \sim \hbar \Omega_{s p}$.

\section{A. Dynamical equations and exact solution}

Under the above assumptions, the time evolution of the system involves only two types of states: (1) $\left|\Psi_{\mathbf{q}}\right\rangle=|G\rangle \otimes\left|1_{\mathbf{q}}\right\rangle$ (QD in the ground state, single SPP $n_{\mathbf{q}}=1, n_{\mathbf{q}^{\prime} \neq \mathbf{q}}=0$ ) and (2) $\left|\Psi_{e}\right\rangle=|E\rangle \otimes|0\rangle$ (QD in the excited state, no plasmons). At any later time the state of the system is a linear combination of these states

$$
|\Psi\rangle=a(t)\left|\Psi_{e}\right\rangle+\sum_{\mathbf{q}} c_{\mathbf{q}}(t)\left|\Psi_{\mathbf{q}}\right\rangle,
$$


where the coefficients $a(t)$ and $c_{\mathbf{q}}(t)$ satisfy the equations

$$
\stackrel{\circ}{1} \dot{a}=\Omega_{e x} a+\sum_{\mathbf{q}} \gamma_{\mathbf{q}} c_{\mathbf{q}}, \quad \stackrel{i}{1} \dot{c}_{\mathbf{q}}=\omega_{\mathbf{q}} c_{\mathbf{q}}+\gamma_{\mathbf{q}}^{*} a .
$$

This system must be solved with the initial conditions $a(0)=1$ and $c_{\mathbf{q}}(0)=0$. Resolving the second equation and substituting the result into the first one we obtain the following integrodifferential (Volterra) equation

$$
\dot{a}=-i \Omega_{e x} a-\int_{0}^{t} K\left(t-t^{\prime}\right) a\left(t^{\prime}\right) d t^{\prime},
$$

with memory kernel

$$
K(t)=\sum_{\mathbf{q}}\left|\gamma_{\mathbf{q}}\right|^{2} \exp \left(-\stackrel{i}{1} \omega_{\mathbf{q}} t\right) .
$$

Its solution is found by Laplace transformation which yields

$$
a(s)=\frac{1}{s+\stackrel{\circ}{i} \Omega_{e x}+K(s)},
$$

where the Laplace transform of the kernel is

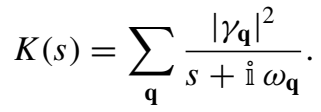

Finally, the time evolution of the system is found by the inverse Laplace transform which is defined as a contour integral in the complex $s$ plane

$$
a(t)=\frac{1}{2 \pi \stackrel{\circ}{1}} \int_{C} a(s) e^{s t} d s,
$$

where the contour $C$ is a vertical line placed to the right of all singularities of $a(s)$.

\section{B. Two relaxation mechanisms}

The dynamical equations (38) conserve the total energy of the system. However, the QD energy decreases with time due to its redistribution over the continuum of SPP modes, which is a typical relaxation mechanism in quantum dissipative systems $[44,45]$. The relaxation rate can be estimated if the kernel $K(t)$ decays faster than the solution $a(t)$ such that one can use the Markov approximation, where $a(t)$ is assumed a slowly changing function and is taken outside the integral in Eq. (39). This yields an exponentially decaying solution

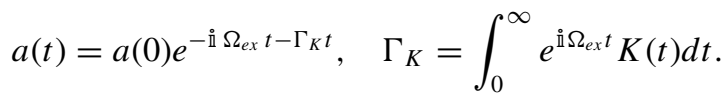

This expression of the decay rate coincides with the result of Fermi's golden rule.

The decay of SPP modes introduces another relaxation mechanism in the QD dynamics, which is not present in the Hamiltonian (35). This mechanism can be taken into account by amending Eq. (35) with the interactions that describe such a decay. Alternatively, one can employ the Linblad formalism with phenomenological decay rates. However, since the frequency dispersion $\omega_{q}$ is already known, we can simply modify $K(t)$ in Eq. (39) by adding the factor $\exp \left(-\omega_{q}^{\prime \prime} t\right)$ with a correct decay rate for each mode. This procedure modifies the Laplace transform of the kernel in Eq. (42) accordingly. The amended dynamics in Eq. (39) accounts for both relaxation mechanisms: the energy redistribution over the continuum and the decay of the modes in the continuum. Obviously, this approach is valid as long as the continuum modes are weakly damped.

\section{Memory kernel and inverse Laplace transform}

An explicit expression for the memory kernel is obtained by substituting $\gamma_{\mathbf{q}}$ from Eq. (33) and $\omega_{q}$ from Eq. (19) into Eqs. (42) and taking the sum (integral) over q. This expression can be simplified by keeping only the leading contribution to the integrand with respect to small $q$. In the integral we neglect the interval $q \lesssim q_{0}$, which introduces an error of the order of $\alpha \ll 1$ (a more accurate estimation of the accuracy of this approximation is found in Appendix). The frequency dispersion is then approximated by the linear dependence in Eq. (19). This yields a simplified expression for the kernel

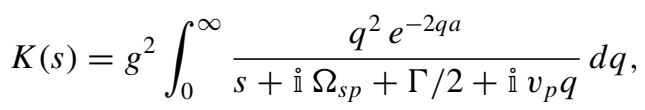

where the effective coupling constant is found as

$$
g^{2}=\frac{3}{\left(1+\varepsilon_{h}\right)^{3 / 2}} \frac{\gamma_{0}}{\alpha^{3} \Omega_{e x}^{3}},
$$

and we used dimensionless quantities $\gamma_{0} \rightarrow \gamma_{0} / \Omega_{p}$ and $\Omega_{e x} \rightarrow \Omega_{e x} / \Omega_{p}$ similar to Eq. (10). The integral in Eq. (45) can be estimated analytically. It is convenient to change the Laplace variable $s$ to

$$
\xi=-\frac{\AA a}{v_{p}}\left(s+\AA \Omega_{s p}+\Gamma / 2\right),
$$

and to introduce scaled constants

$$
\mathfrak{g}=\frac{a}{v_{p}} \tilde{g}, \quad \tilde{g}=\frac{g}{a^{3 / 2}}, \quad \Delta=\frac{a}{v_{p}}(\delta \Omega+\stackrel{i}{1} \Gamma / 2),
$$

where the difference $\delta \Omega=\Omega_{e x}-\Omega_{s p}$ will be referred to as "detuning." The transformation in Eqs. (47) and (48) helps to write the inverse Laplace transform in Eq. (43) in the form

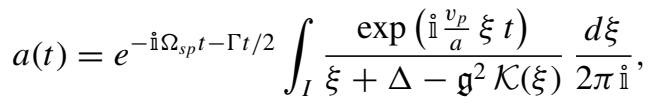

with a simple analytic expression for the kernel

$$
\mathcal{K}(\xi)=\frac{1}{4}-\frac{\xi}{2}+\xi^{2} e^{2 \xi} \Gamma(0,2 \xi),
$$

with $\Gamma(0, x)$ being the incomplete gamma function. The integration contour $I$ in Eq. (49) (see Fig. 3) is obtained from the original contour $\mathcal{C}$ in Eq. (43) by the same change of variables. The tangent angle of the straight line $I$ is equal to the argument of the complex velocity of the SPP excitations, $\theta=-\arg \left[v_{p}\right]$.

The integral in Eq. (49) is evaluated by extending the integral contour $I$ by closing it in the upper half-space of the complex plane $\operatorname{Im}[\xi]>0$, as illustrated in Fig. 3. The contour should bypass singularities (simple poles at $\xi_{i}$ ) and the branch-cut line $J=(-\infty, 0]$ defined by the analytic structure of the function $\mathcal{K}(\xi)$ in Eq. (50). The integral over the closed contour $I$ is equal to a sum

$$
a(t)=-a_{J}(t)-\sum_{i} a_{i}(t),
$$




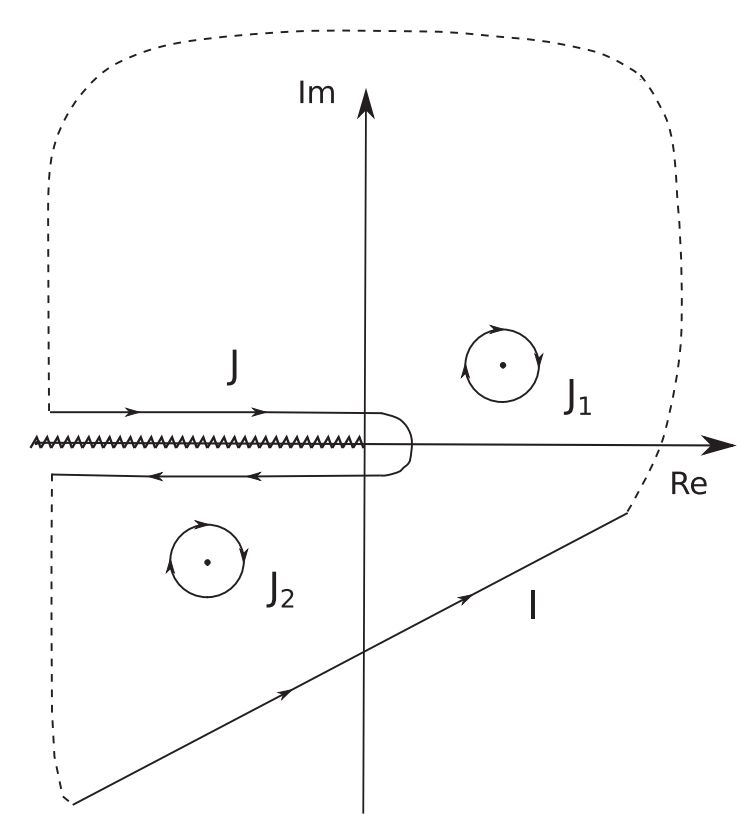

FIG. 3. Illustration of the integration contour for the inverse Laplace transformation in Eq. (49). The line $I$ is obtained from the vertical line of the integral in Eq. (43), its tangent is given as $-\arg \left[v_{p}\right]$. The branch cut of $\mathcal{K}(\xi)$ is indicated by a zigzag line. $J_{i}$ are the poles of the integrand in Eq. (49).

where the branch-cut contribution is

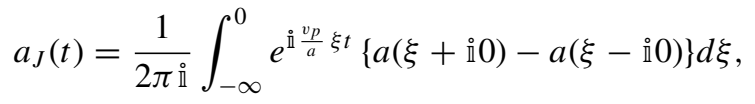

and the pole residues are

$$
a_{i}(t)=-\left[\frac{d a\left(\xi_{i}\right)}{d \xi_{i}}\right]^{-1} \exp \left(\stackrel{\circ}{\frac{v_{p}}{a}} \xi_{i} t\right) .
$$

\section{QD DYNAMICS}

The results of the previous section demonstrate that the QD dynamics depends on three main parameters: detuning $\Delta$, coupling $\mathfrak{g}$, and decay rate $\Gamma$, which contain material parameters $\Omega_{p}, v_{F}$, and $a$ in their scaling. As a prototype we consider a system where the QD is embedded in the semiconductor GaAs and is placed close to a silver sample. Material parameters for silver can be found in Ref. [79].

The dielectric constant of the embedding GaAs material is $\varepsilon_{h} \simeq 11$ (estimated at $\omega=\Omega_{s p}$ ). The energy of bulk plasmons in silver is $\hbar \Omega_{p} \simeq 9.2 \mathrm{eV}$. The energy of surface plasmons, obtained from the condition $\varepsilon=-\varepsilon_{h}$, is $\hbar \Omega_{s p} \simeq 2 \mathrm{eV}$. For the Fermi velocity in bulk silver we take $v_{F} \simeq 1.4 \times 10^{6} \mathrm{~m} / \mathrm{s}$, so that $\alpha=v_{F} / \mathrm{c} \simeq 5 \times 10^{-3}$. The rate of SPP decay due to the finite carrier lifetime in silver at $\omega=\Omega_{s p}$ is estimated as $\Gamma \simeq 0.02 \mathrm{eV}$. This rate is smaller than the rate due to the Landau damping and can be neglected in the time scale of interest. The recombination time of the dot is assumed $\tau \simeq$ $0.5 \mathrm{~ns}$, which yields the corresponding exciton relaxation rate $\gamma_{0} \simeq 2 \times 10^{9} \mathrm{~s}^{-1}$. The QD transition energy $\Omega_{e x}$ is taken as a variable because we intend to consider dynamical patterns at different values of the detuning $\delta \Omega$.
With these material parameters we can estimate the validity interval of the approach and of the approximations made in the analysis of the previous two sections using the results of Appendix. The interval of admissible values of the QD-interface distances is estimated as $a \sim 5-500 \mathrm{~nm}$. At distances $a \gtrsim 500 \mathrm{~nm}$, details of the dispersion at $q \lesssim q_{0}$ become important, while at $a \lesssim 5 \mathrm{~nm}$ the expansion condition $q \ll 1$ (in the dimensionless units) is considerably violated. The validity of the continuous media approximation and the specular reflection assumption breaks at much smaller distances $a \lesssim 1 \mathrm{~nm}$. In principle, the calculations within the continuous models can thus be done also in the interval $1 \mathrm{~nm} \lesssim a \lesssim 5 \mathrm{~nm}$. However, this requires the exact numerical solution of the dispersion equation (11) as well as the analysis of the influence of the shape of the effective potential at the metal boundary on the susceptibility (4) (see Appendix), which although interesting is beyond the scope of this work.

\section{A. Two characteristic times}

Examples of the time evolution of $|a(t)|$, calculated at different detunings $\delta \Omega$, are shown in Figs. 4(a)-4(d) by thick (color) lines. In all panels, the time dependence is initially oscillatory, which is then followed by a longer monotonic decay. One can distinguish three parameters that characterize the dynamical pattern: the frequency of the oscillations $\Omega_{R}$ (Rabi frequency) and two decay rates $\gamma_{R} \gg \gamma_{t}$, that determine the decay of the Rabi oscillations and of the subsequent monotonic relaxation, respectively. A time evolution with two very different rates is a notable feature of this system. We want to consider it in more detail by looking into the analytical structure of $a(\xi)$ in Eq. (41) or in (49).

We note that $a(\xi)$ can have at most two poles. In the case of two poles (the regime of strong coupling) the contribution of the branch-cut integral $J$ is negligible and the time dependence is

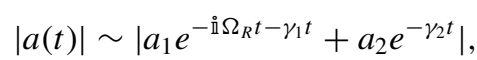

where the frequency $\Omega_{R}$ and the decay rates $\gamma_{i}$ are defined by the poles

$$
\Omega_{R}=\operatorname{Re}\left[\frac{v_{p}}{a}\left(\xi_{1}-\xi_{2}\right)\right], \quad \gamma_{i}=\operatorname{Im}\left[\frac{v_{p}}{a} \xi_{i}\right] .
$$

Equation (54) describes decaying Rabi oscillations with two characteristic decay rates $\gamma_{1}$ and $\gamma_{2}$. The larger rate $\gamma_{R}=$ $\max \left(\gamma_{i}\right)$ describes the decay of the Rabi oscillations, while the smaller one $\gamma_{t}=\min \left(\gamma_{i}\right)$ defines the subsequent long monotonic relaxation. In our examples, the decay of the Rabi oscillations turns out to be much faster than the long-time decay. In fact, Fig. 4 reveals a large difference between the rates that reaches orders of magnitude: $\gamma_{R} / \gamma_{t} \sim 10-100$.

The appearance of two distinct rates can be traced by looking for solutions of $a(\xi)^{-1}=0$ in the limit of large coupling constants. An approximate solution for the poles can be obtained by employing the asymptotic expansion of the kernel for $|\xi| \gg 1$ :

$$
\mathcal{K}(\xi) \simeq \frac{1}{4 \xi}-\frac{3}{8 \xi^{2}}
$$




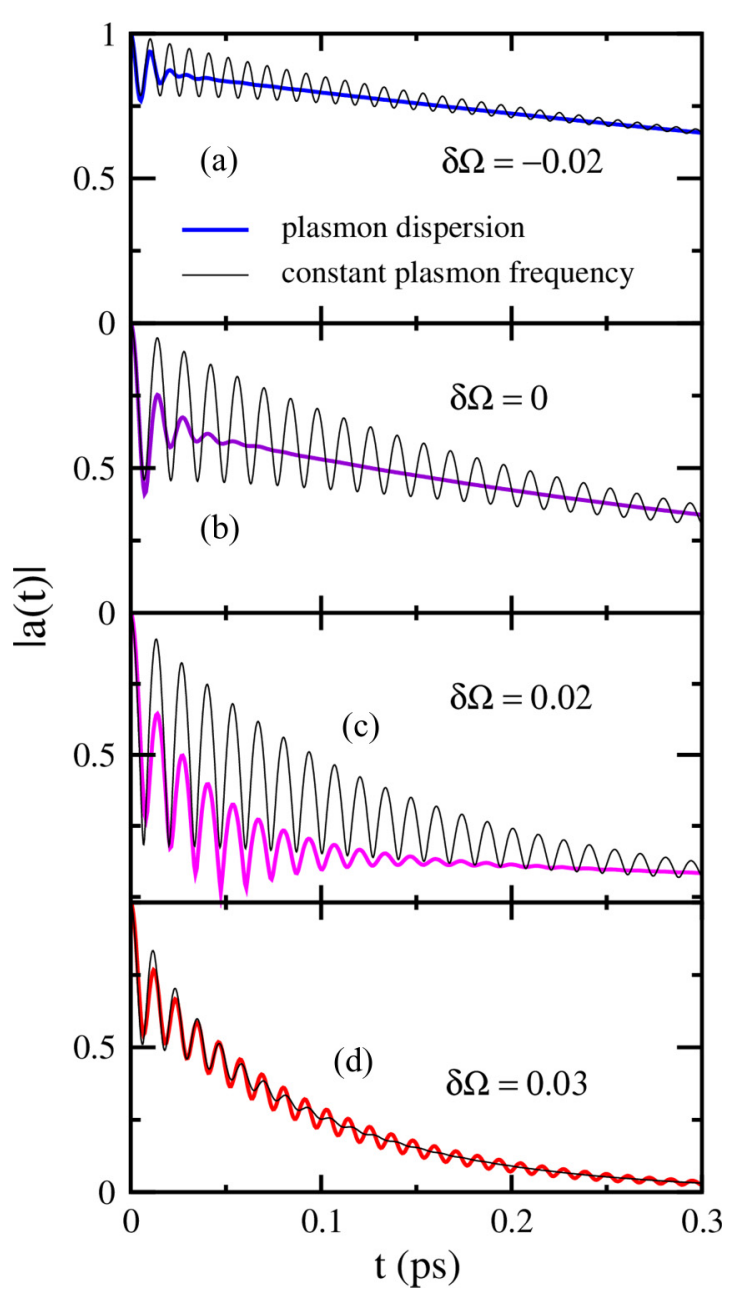

FIG. 4. Examples of the time evolution of the dot. Thick colored lines in panels (a)-(d) represent $|a(t)|$ obtained from Eq. (49) at $\delta \Omega=-0.02,0,0.02,0.03$ (in $\Omega_{p}$ units) for the QD-surface distance $a=10 \mathrm{~nm}$ and other material parameters as discussed in the text. Thin black lines are obtained by neglecting the plasmon dispersion $\omega_{q}=\Omega_{s p}$ and choosing the parameters $\tilde{\Gamma}$ and $\delta \Omega$ to reproduce the frequency $\Omega_{R}$ of the Rabi oscillations and the overall relaxation rate which determines the long-time asymptotic of the QD in the original dynamics (thick lines in this figure).

At negative (real) $\xi$, this expansion should be amended by the imaginary contribution equal to the jump of the kernel on the branch cut: $\operatorname{Im}[K(\xi<0)]=\stackrel{i}{\pi} \xi^{2} e^{2 \xi}$. Substituting this expansion into Eq. (49) we find two poles of the integrand which yield the Rabi frequency

$$
\Omega_{R} \simeq \Omega_{0}-\frac{3}{2} \frac{\delta \Omega}{\tau_{p} \Omega_{0}},
$$

where $\Omega_{0}=\tilde{g}$ and $\tau_{p}=a / v_{p}^{\prime}$, as well as the decay rates

$$
\begin{aligned}
& \gamma_{1} \simeq \frac{3}{4} \frac{\left|v_{p}^{\prime \prime}\right|}{a}\left(1+2 \frac{\delta \Omega}{\Omega_{0}}\right), \\
& \gamma_{2} \simeq \frac{3}{4} \frac{\left|v_{p}^{\prime \prime}\right|}{a}\left(1-2 \frac{\delta \Omega}{\Omega_{0}}\right)+\frac{\pi \Omega_{0}}{4}\left(\Omega_{0} \tau_{p}\right)^{3} e^{-\left(\Omega_{0}+\delta \Omega\right) \tau_{p}} .
\end{aligned}
$$

This result for the rates comprises two contributions of different nature. The first one, found in both $\gamma_{1,2}$, is proportional to $\left|v_{p}^{\prime \prime}\right|$ and appears due to the Landau damping. The second contribution is found only in $\gamma_{2}$ and depends on the new characteristic time $\tau_{p}$, which is equal to the plasmons time of flight over the distance $a$.

We note that the latter contribution is a consequence of the nonzero SPP velocity $v_{p}^{\prime}$ at $q \gtrsim q_{0}$, and thus of the nonlocality in the dielectric response of the metal. When the latter is neglected the frequency dispersion degenerates, $\omega_{q}=\Omega_{s p}$, and the kernel $K(s)$ is given by Eq. (A5) in the Appendix, where the decay rate $\tilde{\Gamma}$ stands for all relaxation channels. The resulting time dependence is defined by two poles

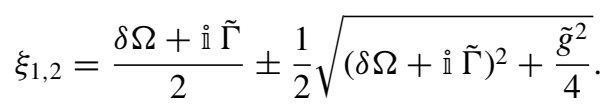

This well-known expression describes the poles that correspond to the dynamical equations for a QD placed in a resonator and coupled to a single electromagnetic mode [72]. The poles for this system reveal a bifurcation point at $\tilde{g}=2 \tilde{\Gamma}$ that separates the strong coupling regime with Rabi oscillations from the weak coupling regime where a monotonic decay is found. Equation (59) yields two relaxation rates $\gamma_{1,2} \simeq$ $\tilde{\Gamma}\left(1 \pm \delta \Omega / \Omega_{0}\right) / 2$, which coincide with the first contribution in Eq. (58), if we take $\tilde{\Gamma}=3\left|v_{p}^{\prime \prime}\right| /(2 a)$. However, the second contribution to $\gamma_{2}$ in Eq. (58) cannot be reproduced without accounting for the nonlocality in the dielectric response.

The difference between the decay rates in Eq. (59) is determined by the relative detuning $\delta \Omega / \Omega_{0}$. This quantity is small (or zero) and thus Eq. (59) cannot explain the large differences between the rates seen in Fig. 4. An attempt to reproduce the dynamics induced by the Lindhard permittivity function using the results for the local response, with $\delta \Omega, \tilde{\Gamma}$, and $g$ playing the role of fitting parameters, does not lead to a satisfactory agreement. This is clearly seen in Fig. 4, where the best fits (thin black lines) are plotted alongside the original time dependencies (color thick lines).

When the coupling strength decreases one or both poles of $a(\xi)$ move toward the branch cut and at some critical value of the coupling strength one of them disappears. In this case, the contribution of the integral along the branch cut in Eq. (52) becomes comparable with that of the remaining pole. However, for a wide range of coupling constants the dynamical pattern does not differ qualitatively from the case of two poles. The reason is that the dependence of $a(\xi)$ along the branch cut is similar to the Lorenzian dependence (this happens if the pole is not too far from the branch cut in the unphysical sheet). In this case, the integral yields a time evolution which resembles a simple exponential dependence, as would result from the pole residue. We note that for the parameters used to plot Figs. 4(a)-4(c) the system has a single pole and a Lorenziantype dependence of $a(\xi)$ along the branch cut. The second pole appears only at $a \lesssim 5 \mathrm{~nm}\left(g \propto a^{-1 / 2}\right)$, where the applicability of the employed theoretical approach becomes questionable. Nevertheless, the conclusion on the two distinct decay times remains valid in a broad range of coupling strengths.

Finally, we note that the smaller of the two decay times $\gamma_{t}$ can be estimated without the full analysis of the dynamics, e.g., by a simple analysis of the energy loss rate due to the dipole dot-plasmon coupling, as is done, in particular in Refs. [5052]. Calculations based on such estimations are more accurate 
far from the resonance, where the ultrafast dynamics is suppressed. In Sec. IV D we determine the dependence on the distance $a$ and demonstrate that the asymptotic dependence of $\gamma_{t}$ coincides with the earlier results.

\section{B. Power-law asymptote}

When the coupling constant $g$ decreases or the detuning becomes larger, the dependence $a(\xi)$ ceases to be a Lorenziantype function. In this case, the branch-cut integral $a_{J}(t)$ in Eq. (52) gives a power-law long-time asymptotic $t^{-\alpha}$. This qualitative change is most visibly manifested in a much slower decay of the Rabi oscillations and not in the longer time asymptotic of $|a(t)|$. The latter follows from the fact that similarly to Eq. (54), the time dependence comprises two contributions, the exponential and the power-law functions

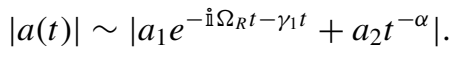

It turns out that the coefficient $a_{2}$ is much smaller than $a_{1}$ so that the power-law contribution is smaller than the exponential one at intermediate times. As long as this condition holds, the decay of the oscillations is governed by the power law as can be seen by expanding the square root which is contained in the modulus in Eq. (60) with respect to the parameter $a_{2} / a_{1} e^{\gamma_{1} t} t^{-\alpha}$ which is small for not too long times. Consequently, the oscillations persist much longer than in the case when both contributions are exponential. At longer times, the power-law contribution in Eq. (60) becomes dominant. However, in this limit the dynamics is suppressed by the common exponential factor $\exp (-\Gamma t / 2)$ in Eq. (49), such that in practice the power-law dependence does not reveal itself in the long-time asymptote.

The time evolution in this regime is illustrated in Fig. 4(d) where the time dependence is calculated at $\delta \Omega=0.03$. One can see that, although the overall relaxation is faster [compared with Figs. 4(a)-4(c)], the decay of the Rabi oscillations slows down, so that the oscillations are visible at much longer times.

The power-law time contribution can be estimated analytically from Eq. (52) by looking at the asymptote of its integrand at $\xi \rightarrow 0$. Expanding $\mathcal{K}(\xi)$ at small $\xi$ we obtain

$$
K(\xi) \simeq \frac{1}{4}-\frac{\xi}{2}-\xi^{2} \ln \left(2 e^{\gamma} \xi\right) .
$$

With the help of this expansion, Eq. (52) is rewritten as

$$
a_{J}(t) \simeq-\int_{-\infty}^{0} \frac{\mathfrak{g}^{2} \xi^{2} \exp \left(\mathfrak{i} \frac{v_{p}}{a} \xi t\right)}{\left(\xi+\Delta-\mathfrak{g}^{2} / 4\right)^{2}+\pi^{2} \mathfrak{g}^{2} \xi^{4}} d \xi .
$$

Its long-time asymptotic is then found by substituting $x=\xi t$ and expanding the integrand with respect to large $t$ which yields

$$
a_{J}(t) \simeq \frac{2 a^{3} \mathfrak{g}^{2}}{b^{3}\left(\Delta-\mathfrak{g}^{2} / 4\right)^{2}} \frac{1}{t^{3}} .
$$

This expression formally diverges at $\Delta=\mathfrak{g}^{2} / 4$. This means that close to this point the next order contributions of the expansion in Eq. (62) must be taken into account giving even a slower decaying function $a_{J}(t) \sim t^{-1}$.

We stress again that the power-law dependence is a direct consequence of the linear dependence of the plasmon frequency dispersion at larger $q$ that appears due to the nonlocality of the metal permittivity function. The power-law decay cannot appear for the Drude permittivity model.

\section{Dependence on detuning}

According to Eq. (49), the QD dynamics depends on two parameters: the (scaled) detuning $\Delta$ and the coupling constant $\mathfrak{g}$ (we assume that $\Gamma$ is negligibly small). As demonstrated above, a typical dynamical pattern is an oscillating relaxation characterized by two decay rates $\gamma_{R}$ and $\gamma_{t}$ (the latter is defined only for the exponential decaying case) and the Rabi frequency $\Omega_{R}$. Here, we investigate the dependence of $\Omega_{R}$ and $\gamma_{t, R}$ on the detuning $\Delta(\delta \Omega)$. The parameters $\Omega_{R}$ and $\gamma_{t, R}$ are obtained directly from the numerical time dependence of $|a(t)|$ by fitting it with the approximate expression (54).

The results are shown in two panels in Fig. 5. For a convenient comparison with the time evolution in Fig. 4, the values of the detuning $\delta \Omega=-0.02,0,0.02,0.03$, used to obtain the dynamics in panels Figs. 4(a)-4(d), respectively, are marked by (colored) points in Fig. 5. We note that $\gamma_{1}$ and $\gamma_{2}$ in Fig. 5(b) reflect the structure of the integrand in Eq. (49) and are thus continuous functions of the system parameters. Since $\gamma_{t, R}$ are defined as the minimum/maximum of $\gamma_{1,2}$, the
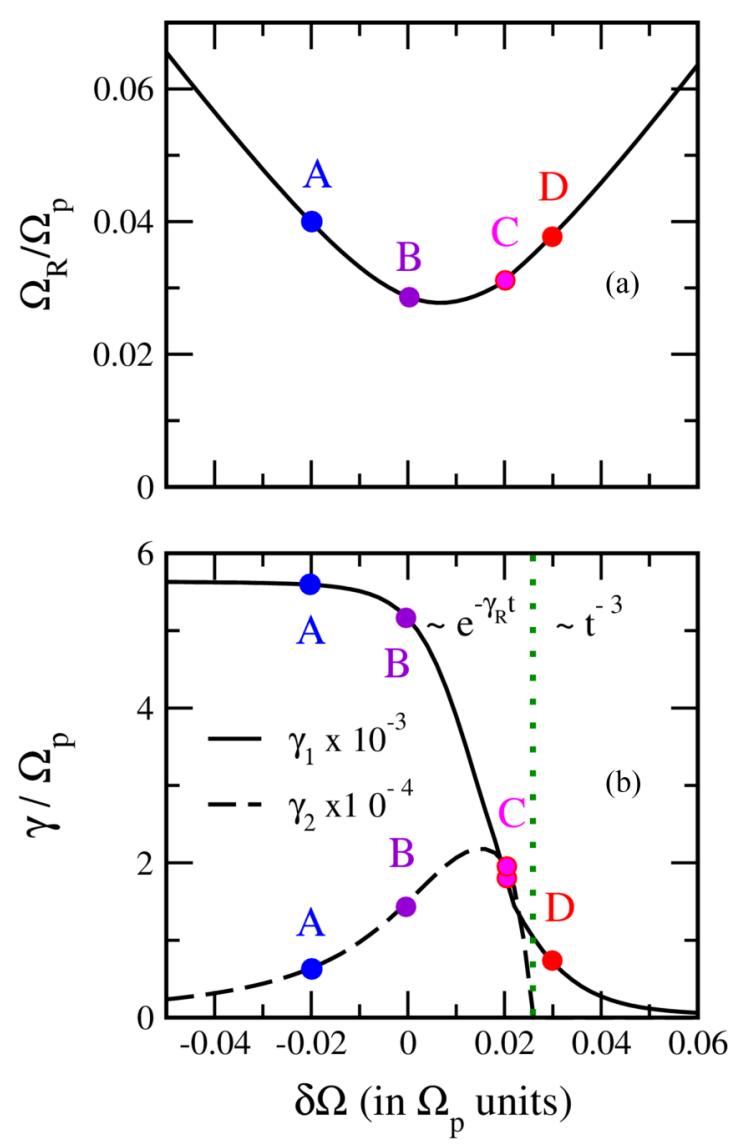

FIG. 5. Dependence of the characteristics of the system dynamics on the detuning $\delta \Omega=\Omega_{e x}-\Omega_{s p}$. (a) Frequency of the Rabi oscillations $\Omega_{R}$. (b) Two decay rates $\gamma_{1,2}$. All quantities are shown in the scaled units of Eq. (10). Circles A-D (colored) mark the detunings $\delta \Omega$ used in the calculation of the time evolution in Figs. 4(a)-4(d), respectively. The QD-surface distance is $a=10 \mathrm{~nm}$, other material parameters are as discussed in the text. 
correspondence $\gamma_{t, R} \leftrightarrow \gamma_{1,2}$ may change as is seen in Fig. 5(b), where it interchanges at $\delta \Omega \sim 0.02$.

The dependence $\Omega_{R}(\delta \Omega)$, shown in Fig. 5(a), is well described by the parabolic function

$$
\Omega_{R} \sim \sqrt{\left(\delta \Omega-\delta \Omega_{0}\right)^{2}+\tilde{g}^{2} / 4},
$$

with the minimum at $\delta \Omega_{0}>0$. This is similar to the wellknown result for the Rabi frequency of a QD coupled to a single resonator mode [72], obtained from Eq. (59), where the additional shift $\delta \Omega_{0} \propto a^{-1}$ reflects the position of the maximal value of the spectral weight of the QD-SPP coupling.

In contrast to $\Omega_{R}(\delta \Omega)$, the decay rates $\gamma_{i}(\delta \Omega)$ [Fig. 5(b)] are nonsymmetric functions of $\delta \Omega$. At $\delta \Omega<0$ the smallest rate $\gamma_{t}=\gamma_{2}$, that defines a slow monotonic relaxation, corresponds to the pole $J_{2}$ in the upper half-plane in Fig. 3. The larger rate $\gamma_{1}$ is determined by either the pole $J_{1}$ in the lower half-space or by the branch cut $J$. It is important that with increasing $\delta \Omega$ the rates $\gamma_{1}$ and $\gamma_{2}$ shift toward each other, so that the decay of the Rabi oscillations slows down while the overall relaxation becomes faster. At $\delta \Omega \gtrsim 0.015$, the rate $\gamma_{2}(\delta \Omega)$ becomes a decreasing function. It reaches zero at $\delta \Omega \simeq 0.025$ where the dynamics changes qualitatively. When the detuning exceeds this value $a_{J}(t)$ has the power-law long-time asymptote $\propto t^{-3}$ (the exponential decay rate is formally zero). This change is manifested in the longer visibility of the Rabi oscillations as illustrated in Fig. 4(d).

\section{Dependence on the QD-surface distance}

The second parameter that determines the system dynamics is the coupling strength $\mathfrak{g}$, which according to Eq. (48) is controlled by the QD-surface distance $a\left(\tilde{g} \propto a^{-1 / 2}\right)$. Unlike the QD dipole moment, this distance is easily controlled in experiments.

The Rabi frequency $\Omega_{R}$ as a function of $a$ is shown in Fig. 6. We note that the obtained dependence is also well described by Eq. (64). The calculations at $\delta \Omega=-0.02,0$, labeled as $\mathrm{A}$ and $\mathrm{B}$ in Fig. 6, correspond to the similarly labeled curves

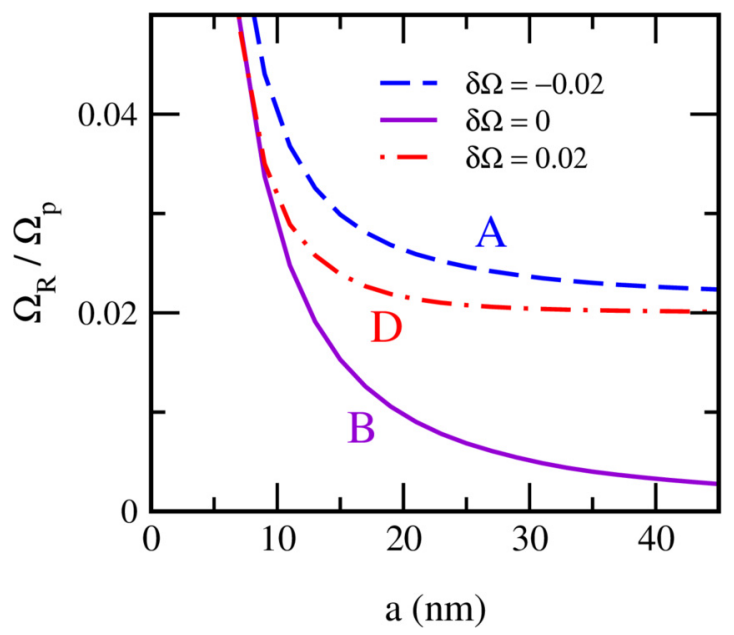

FIG. 6. Frequency of the Rabi oscillations $\Omega_{R}$ as a function of the QD-surface distance $a$, calculated at $\delta \Omega=-0.02,0,0.02$ (in $\Omega_{p}$ units), labeled as A, B, and D, that correspond to the time evolution in panels Figs. 4(a), 4(b), and 4(d).
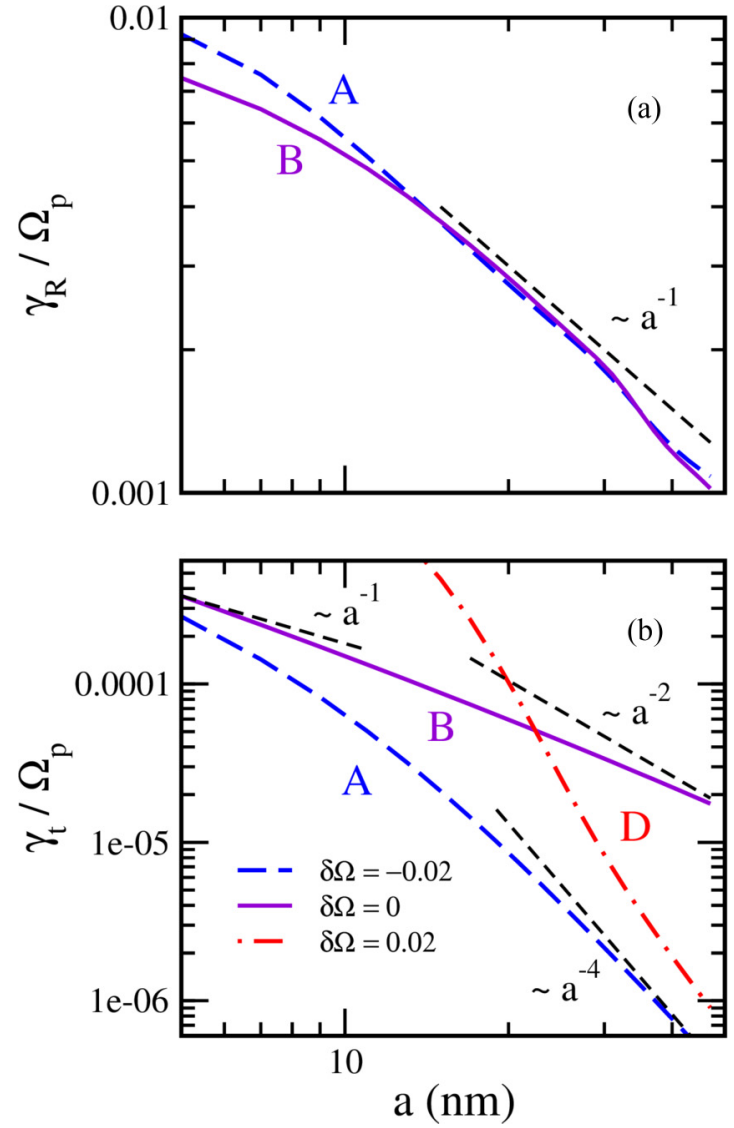

FIG. 7. Log-log plot of the decay rates $\gamma_{R}$ (a) and $\gamma_{t}$ (b) as functions of the QD-surface distance $a$ calculated at $\delta \Omega=-0.02,0,0.02$ (in $\Omega_{p}$ units). The labeling of the curves corresponds to that in Fig. 6. The case $\delta \Omega=0.02$ (D) is absent for $\gamma_{R}$ in (a) because the corresponding contribution has a power-law asymptotic.

and points in the other figures. The line D in Fig. $6(\delta \Omega=$ 0.02 ) corresponds to results in Fig. 4(d) and at point D in Fig. 5, that are both calculated for a QD-surface distance $a \gtrsim$ $15 \mathrm{~nm}$. However, at smaller distances $a \lesssim 15 \mathrm{~nm}$, the line D in Fig. 6 corresponds to Fig. 4(c) and to point $\mathrm{C}$ in Fig. 5. This interchange follows from the fact that the scaled detuning depends on the distance as $\Delta \propto a$ and, therefore, variations in $a$ lead to respective changes in $\delta \Omega$.

The rates $\gamma_{R}$ and $\gamma_{t}$ are shown, respectively, in panels (a) and (b) in the log-log Fig. 7. The calculations at $\delta \Omega=$ $-0.02,0,0.02$ are labeled as A, B, and D. Figure 7(a) does not show line $\mathrm{D}(\delta \Omega=0.02)$ since it exhibits a power-law asymptotic.

Both $\gamma_{t}$ and $\gamma_{R}$ are decreasing functions of $a$. In the limits of small and large $a$, the smallest rate $\gamma_{t}$ can be approximated by power-law dependencies of the type $\gamma_{i} \propto a^{-v}$. In particular, at small $a$ and large coupling constants Eq. (58) gives $\gamma_{t} \sim a^{-1}$, which is shown by a dashed line for small $a$ 's in Fig. 7(b). One sees that the asymptotic estimate is close to the numerical results at $\delta \Omega=-0.02,0$ (lines $\mathrm{A}$ and $\mathrm{B}$ ). It must be noted, however, that this asymptote is achieved at very small $a$, which is beyond the validity interval of the approach $(a \gtrsim 5 \mathrm{~nm})$. At $\delta \Omega=0.02$ (line D) this asymptote could not be achieved. 
In the opposite limit of large $a$, the coupling constant $\mathfrak{g} \propto$ $a^{-1 / 2}$ is small. However, since the scaled detuning $\Delta \propto a$ is large in this limit, the asymptotic in Eq. (56) can be used for the estimations of the pole. From this one estimates the asymptote of the smallest rate $\gamma_{t}$ as

$$
\gamma_{t} \simeq \frac{3}{8} \frac{\left|v_{p}^{\prime \prime}\right|}{\delta \Omega^{2}} \frac{g^{2}}{a^{4}} .
$$

As mentioned earlier at the end of Sec. IV A, the results of the calculations for $\gamma_{t}$ far from the resonance should coincide with previous calculations of the dot relaxation rate, based on the estimate for the QD decay rate from the plasmon energy loss as described by the imaginary part of the frequency dispersion $\omega_{q}^{\prime \prime}$. Indeed, the asymptote $a^{-4}$ obtained in Eq. (65) coincides with the earlier results in this limit, derived in particular in Ref. [51].

As seen in Fig. 7(b), where Eq. (65) is shown by the dashed lines at larger $a$, the asymptote $\gamma_{t} \sim a^{-4}$ is in good agreement with the numerical results at $\delta \Omega \neq 0$. Finally, at $\delta \Omega=0$ the analytic estimation yields $\gamma_{t} \sim a^{-2}$, however, it is achieved at much larger $a$ than shown in Fig. 7.

The asymptotes of $\gamma_{R}$ are not so simple, which can be seen from the approximation in Eq. (58). Figure 7(a) reveals a more complicated dependence $\gamma_{R}(a)$. At small $a$ the additional contribution disappears and one obtains $\gamma_{R} \sim a^{-1}$. However, the numerical curves in Fig. 7(a) are not close to these asymptotes, showing instead a more complicated pattern.

\section{SUMMARY}

This work presents an investigation of the quantum dynamics of a QD in the vicinity of a metallic surface. The dynamics is induced by the electromagnetic interaction between the QD and SPP excitations localized near the metal-insulator interface. The calculations are done using a continuous media model with linear electromagnetic response for both metal and insulator. The approach takes into account the nonlocality of the electromagnetic response of the metal, given by the Lindhard function which is modified for the half-space geometry. An analysis of the validity of the model and the used approximations indicates that the conclusions can be trusted in a wide range of parameters. In particular, for a typical metal the interval of acceptable dot-surface distances is estimated as $5 \lesssim a \lesssim 500 \mathrm{~nm}$.

Calculations of the SPP modes demonstrate that the nonlocality significantly modifies their frequency dispersion. It becomes linear at larger wave vectors, so that plasmons in this interval propagate with a finite velocity estimated as $\sim v_{F}$. It is also shown that the damping of SPP modes is controlled by the Landau damping in the metal. Both effects lead to notable changes in the system dynamics.

The time evolution of the system is calculated for the case where the QD is initially prepared in its excited state while SPP excitations are absent. The dynamics is shown to comprise two components: a rapidly decaying oscillation followed by a much slower monotonic decay at longer times. The appearance of two relaxation rates that differ by large amounts is shown to be another consequence of the nonlocality of the electromagnetic response.
The system dynamics is controlled by two main parameters: (i) the detuning between the surface plasmon frequency derived from the Drude model and the dot transition frequency and (ii) the coupling strength. The latter can be adjusted by the distance between the dot and the surface. The analysis reveals specific dependencies of the dynamical characteristics on these parameters.

Finally, it is found that under certain conditions, one of the contributions to the relaxation dynamics has a power-law longtime asymptote. Although at longer times this contribution is suppressed by other relaxation channels, it leads to a considerably reduced damping of the Rabi oscillations.

\section{ACKNOWLEDGMENTS}

The authors thank M. Lippitz, L. Meissner, and M. Brandstetter for fruitful discussions. This research was supported by funding from the European Comission (EU's Seventh Framework Programme - FP7, 2007-2013) under the Grant Agreement No. PCOFUND-GA-2009-246542. I.A.L. acknowledges support from the Foundation for Science and Technology (FCT, Portugal) and M.D.C. acknowledges support of the Belgian Science Policy (BELSPO Back to Belgium Grant).

\section{APPENDIX: ACCURACY OF THE APPROXIMATIONS}

The calculations in this work involve several approximations. The first one is the continuous media model in the calculations of SPP modes, in particular, the electromagnetic response of the metal. This approximation neglects changes of the spatial distribution of the charge carriers in the vicinity of the interface. The corresponding width where such changes are important can be estimated as $2 \pi k_{F}^{-1}$, and this approximation is obviously not valid at smaller dot-interface distances. For parameters of silver and gold, one estimates $2 \pi k_{F}^{-1} \approx 0.5 \mathrm{~nm}$, which is close to the interatomic distance in these metals $a_{m} \approx 0.3 \mathrm{~nm}$. This sets the lowest limit of the validity interval, further limits are determined by the approximations used in the calculations within the model.

The lower limit for $a$ follows from the applicability of the linear approximation for the dispersion (19). Taking the validity interval for this expansion as $q \ll 1$ and the exponential cutoff $\exp (-2 q a)$, that appears in the expression of the QD-SPP coupling constants, one obtains the validity interval for dot-surface distances as $a \gg 1$, or $a \gg v_{F} / \Omega_{p}$ in the original dimensional units. With the material parameters discussed in the text, this yields the lowest limit of the validity interval $v_{F} / \Omega_{p} \approx 0.7 \mathrm{~nm}$. In practice, however, $a$ must be larger than this limit. The accuracy of the linear approximation (19) is estimated as $\sim 3 \%$ for $1 / q=5 \mathrm{~nm}$, which implies a similar accuracy for $a \approx 5 \mathrm{~nm}$.

We note that the formalism developed in this work can be in principle extended to the interval of smaller distances, $0.5 \mathrm{~nm} \lesssim a \lesssim 5 \mathrm{~nm}$. However, in this interval the linear approximation for the dispersion is not applicable and Eq. (11) must be solved numerically. In addition, at such small distances the size of the dot becomes larger than $a$, which requires more elaborate calculations of the coupling constants than used to obtain Eq. (33). However, other components of the model may become questionable in this interval. In particular, at 
such distances the influence of the interface can distort the wave functions of the metal carriers, which can break the SRM assumptions and lead to considerable modifications in the dielectric permittivity, which is beyond the scope of this work.

The upper limit for distance $a$ is defined by the approximations used for the calculation of the memory kernel in Eq. (45), which neglects the interval $q \lesssim q_{0}=\alpha \sqrt{\varepsilon_{h} /\left(1+\varepsilon_{h}\right)}$ of the polariton SPP states. The correct dispersion in this interval is substituted by extending its linear approximation in Eq. (19) to all $q$. In order to estimate the validity of this approximation, we note that the exponential cutoff factor selects the most contributing interval in the integration in Eq. (45) to $q \gtrsim q_{0}$. This gives an estimate for the validity interval $a \lesssim 1 / q_{0} \sim 1 / \alpha$. With the material parameters used in the calculations, we obtain $a \lesssim 100 \mathrm{~nm}$.

A more accurate estimation of the allowed interval of $a$ can be obtained by calculating corrections to the memory kernel $K(s)$ that are given by the SPP states in the interval $q \lesssim q_{0}$. In this interval, the frequency dispersion is obtained by solving Eq. (15) with $\beta(\omega)=0$ which yields the leading contribution in the form

$$
\omega_{q \lesssim q_{0}}=\frac{q}{\alpha \sqrt{\varepsilon_{h}}}\left(1-\frac{1}{2} \frac{q^{2}}{\alpha^{2}}\right) .
$$

The electric field for those SPP modes is given by Eq. (31) with the dispersion, defined in the above equation, and the normalization constant, obtained as

$$
Z_{q \lesssim q_{0}}=\frac{\pi S}{4} \frac{\alpha}{\varepsilon_{h} q^{2}} .
$$

The so-obtained expression for the electric field of the SPP modes is then used to calculate the coupling constants. Corrections to the memory kernel are given by the integral over wave vectors $q$ as in Eq. (45), but with the upper limit $q_{0}$. This limit is conveniently carried out by introducing an exponential cutoff $\exp \left(-q / q_{0}\right)$. This procedure yields a corrective contribution for the kernel in the form

$$
\delta K_{1}(\xi)=\mathfrak{g}^{2} \frac{\alpha^{3} a^{2} v_{p}}{\sqrt{1+\varepsilon_{h}}} \mathcal{K}_{1}\left(\frac{v_{p}}{a} \xi-1\right)
$$

where

$$
\mathcal{K}_{1}(x)=6+\xi\left(-2+\xi-\xi^{2}\right)+\xi^{4} e^{\xi} \Gamma(0, \xi) .
$$

Comparing this with the main contribution to the memory kernel in Eqs. (49) and (50) and taking into account that $\mathcal{K}(x) \sim \mathcal{K}_{1}(x)$ and that $v_{p} \sim 1$, one obtains the condition that the correction is small, as $\alpha^{3} a^{2} / \sqrt{1+\varepsilon_{h}} \ll 1$. This result helps to estimate the validity interval of the calculations as $a \lesssim \alpha^{-3 / 2} \sqrt{1+\varepsilon_{h}}$, which with the numerical parameters used in the calculations yields the validity interval $a \lesssim 0.5 \mu$. At larger distances, the corrections due to small wave vectors must be taken into account. Furthermore, the upper restriction on the distance to the dot is also related to the time light needs to propagate between the dot and the surface. At larger $a \gtrsim$ $0.5 \mu$, this time becomes long enough to influence the system dynamics and this invalidates the quasistatic approximation, where the delay due to the finite flight time is neglected.

We next note that, unlike the interval $q \lesssim q_{0}$, the linear dispersion $\omega_{q}$ at $q \gtrsim q_{0}$ cannot be neglected, so that one cannot approximate $\omega_{q}$ by a constant $\Omega_{s p}$. Indeed, with this approximation the memory kernel is found to be

$$
K(s)=\frac{g^{2}}{4 a^{3}} \frac{1}{s+i \Omega_{s p}+\tilde{\Gamma}},
$$

where $\tilde{\Gamma}$ represents all relaxation mechanisms. The discussion in Sec. IV demonstrates that the dynamics obtained with Eq. (A5) differs qualitatively from Eq. (49).

Finally, in the calculations of the memory kernel in Eq. (45), we neglected the next-to-leading order corrections $\propto q$ that appear in the coupling constants in Eq. (33). Taking those corrections into account, we obtain corrections to the memory kernel as

$$
\delta K_{2}(\xi)=-\frac{\mathfrak{g}^{2}}{a} \frac{\varepsilon_{h}\left(4+\varepsilon_{h}\right)}{\left(1+\varepsilon_{h}\right)^{3 / 2}} \mathcal{K}_{2}(\xi)
$$

with

$$
\mathcal{K}_{2}(\xi)=\frac{1}{4}\left\{1+\xi(-1+2 \xi)-4 \xi^{3} e^{2 \xi} \Gamma(0,2 \xi)\right\} .
$$

Noting that the functions $\mathcal{K}_{2}(\xi) \sim \mathcal{K}(\xi)$ are of the same order, we get the interval $a \gtrsim \varepsilon_{h}\left(4+\varepsilon_{h}\right) /\left(1+\varepsilon_{h}\right)^{3 / 2}$, where such corrections can be neglected. This yields the validity condition $a \gtrsim 5 \mathrm{~nm}$ for the assumed material parameters. Summarizing the results in this appendix we conclude that the analysis in this work is valid for distances 5-500 nm.
[1] H. Raether, Surface Plasmons (Springer, Berlin, 1988).

[2] S. A. Maier, Plasmonics: Fundamentals and Applications (Springer, New York, 2007).

[3] M. L. Brongersma and P. G. Kik (Eds.), Surface Plasmon Nanophotonics, Springer Series in Optical Science Vol. 131 (Springer, Dordrecht, 2007).

[4] R. E. Slusher, L. W. Hollberg, B. Yurke, J. C. Mertz, and J. F. Valley, Phys. Rev. Lett. 55, 2409 (1985).

[5] A. F. Koenderink, Nano Lett. 9, 4228 (2009).

[6] Y. Chen, P. Lodahl, and A. F. Koenderink, Phys. Rev. B 82, 081402 (2010).
[7] C. H. Gan, J. P. Hugonin, and P. Lalanne, Phys. Rev. X 2, 021008 (2012).

[8] N. P. de Leon, B. J. Shields, C. L. Yu, D. E. Englund, A. V. Akimov, M. D. Lukin, and H. Park, Phys. Rev. Lett. 108, 226803 (2012).

[9] R. F. Oulton, V. J. Sorger, T. Zentgraf, R.-M. Ma, L. Dai, G. Bartal, and X. Zhang, Nature (London) 461, 629 (2009).

[10] R-M. Ma, R. F. Oulton, V. J. Sorger, and X. Zhang, Laser Photon. Rev. 7, 1 (2012).

[11] D. J. Bergman and M. I. Stockman, Phys. Rev. Lett. 90, 027402 (2003). 
[12] M. A. Noginov, G. Zhu, A. M. Belgrave, R. Bakker, V. M. Shalaev, E. E. Narimanov, S. Stout, E. Herz, T. Suteewong, and U. Wiesner, Nature (London) 460, 1110 (2009).

[13] E. S. Andrianov, N. M. Chtchelkatchev, and A. A. Pukhov, Phys. Rev. A 88, 053826 (2013).

[14] O. Hess, J. B. Pendry, S. A. Maier, R. F. Oulton, J. M. Hamm, and K. L. Tsakmakidis, Nat. Mater. 11, 573 (2012).

[15] V. Giannini, A. I. Fernández-Dominguez, S. C. Heck, and S. A. Maier, Chem. Rev. 111, 3888 (2011).

[16] E. Eizneer and T. Ellenbogen, Appl. Phys. Lett. 104, 23301 (2014).

[17] A. O. Govorov and I. Carmeli, Nano Lett. 7, 620 (2007).

[18] D. E. Chang, A. S. Sørensen, E. A. Demler, and M. D. Lukin, Nat. Phys. 3, 807 (2007).

[19] P. Kolchin, R. F. Oulton, and X. Zhang, Phys. Rev. Lett. 106, 113601 (2011).

[20] R. Frank, Phys. Rev. B 85, 195463 (2012).

[21] D. E. Chang, A. S. Sørensen, P. R. Hemmer, and M. D. Lukin, Phys. Rev. Lett. 97, 053002 (2006).

[22] D. E. Chang, A. S. Sørensen, P. R. Hemmer, and M. D. Lukin, Phys. Rev. B 76, 035420 (2007).

[23] F. Verstraete, M. M. Wolf, and J. I. Cirac, Nat. Phys. 5, 633 (2009).

[24] M. S. Tame, K. R. McEnery, S. K. Özdemir, J. Lee, S. A. Maier, and M. S. Kim, Nat. Phys. 9, 329 (2013).

[25] J. A. Schuller, E. S. Barnard, W. Cai, Y. C. Jun, J. S. White, and M. L. Brongersma, Nat. Mater. 9, 193 (2010).

[26] J. Bellessa, C. Bonnand, J. C. Plenet, and J. Mugnier, Phys. Rev. Lett. 93, 036404 (2004).

[27] Y. Sugawara, T. A. Kelf, J. J. Baumberg, M. E. Abdelsalam, and P. N. Bartlett, Phys. Rev. Lett. 97, 266808 (2006).

[28] T. K. Hakala, J. J. Toppari, A. Kuzyk, M. Pettersson, H. Tikkanen, H. Kunttu, and P. Törmä, Phys. Rev. Lett. 103, 053602 (2009).

[29] D. E. Gomes, K. C. Vernon, P. Mulvaney, and T. J. Devis, Nano Lett. 10, 274 (2010).

[30] D. Pines, Phys. Rev. 92, 626 (1953).

[31] J. Hopfield, Phys. Rev. 112, 1555 (1958).

[32] R. H. Ritchie, Phys. Rev. 106, 874 (1957).

[33] J. M. Elson and R. H. Ritchie, Phys. Rev. B 4, 4129 (1971).

[34] M. Mivelle, T. S. van Zanten, L. Neumann, N. F. van Hulst, and M. F. Garcia-Parajo, Nano Lett. 12, 5972 (2012).

[35] P. Vasa, W. Wang, R. Pomraenke, M. Lammers, M. Maiuri, C. Manzoni, G. Cerullo, and C. Lienau, Nat. Photonics 7, 128 (2013).

[36] E. M. Purcell, Phys. Rev. 69, 681 (1946).

[37] V. I. Kukushkin, I. M. Mukhametzhanov, I. V. Kukushkin, V. D. Kulakovskii, I. V. Sedova, S. V. Sorokin, A. A. Toropov, S. V. Ivanov, and A. S. Sobolev, Phys. Rev. B 90, 235313 (2014).

[38] P. Vasa, R. Pomraenke, S. Schwieger, Yu. I. Mazur, V. Kunets, P. Srinivasan, E. Johnson, J. E. Kihm, D. S. Kim, E. Runge, G. Salamo, and C. Lienau, Phys. Rev. Lett. 101, 116801 (2008).

[39] A. Neogi, C.-W. Lee, H. O. Everitt, T. Kuroda, A. Tackeuchi, and E. Yablonovitch, Phys. Rev. B 66, 153305 (2002).

[40] K. Okamoto, I. Niki, A. Scherer, Y. Narukawa, T. Mukai, and Y. Kawakami, Appl. Phys. Lett. 87, 071102 (2005).

[41] K. J. Russell, T.-L. Liu, S. Cui, and E. L. Hu, Nat. Photonics 6, 459 (2012).
[42] L. Dominici, D. Colas, S. Donati, J. P. Restrepo Cuartas, M. De Giorgi, D. Ballarini, G. Guirales, J. C. López Carreño, A. Bramati, G. Gigli, E. del Valle, F. P. Laussy, and D. Sanvitto, Phys. Rev. Lett. 113, 226401 (2014).

[43] C. M. Galloway, P. G. Etchegoin, and E. C. Le Ru, Phys. Rev. Lett. 103, 063003 (2009).

[44] A. J. Leggett, S. Chakravarty, A. T. Dorsey, M. P. A. Fisher, A. Garg, and W. Zwerger, Rev. Mod. Phys. 59, 1 (1987).

[45] U. Weiss, Quantum Dissipative Systems (World Scientific, Singapore, 1999).

[46] T. V. Teperik, P. Nordlander, J. Aizpurua, and A. G. Borisov, Phys. Rev. Lett. 110, 263901 (2013).

[47] A. I. Fernández-Domínguez, A. Wiener, F. J. García-Vidal, S. A. Maier, and J. B. Pendry, Phys. Rev. Lett. 108, 106802 (2012).

[48] F. J. G. de Abajo, J. Phys. Chem. C 112, 17983 (2008).

[49] L. D. Landau and E. M. Lifshitz, Electrodynamics of Continuous Media (Pergamon, Oxford, 1960).

[50] G. W. Ford and W. H. Weber, Phys. Rep. 113, 195 (1984).

[51] I. A. Larkin, M. I. Stockman, M. Achermann, and V. I. Klimov, Phys. Rev. B 69, 121403(R) (2004).

[52] I. A. Larkin and M. I. Stockman, Nano Lett. 5, 339 (2005).

[53] K. L. Kliewer and R. Fuchs, Phys. Rev. 172, 607 (1968).

[54] B. B. Dasgupta and R. Fuchs, Phys. Rev. B 24, 554 (1981).

[55] R. Fuchs and F. Claro, Phys. Rev. B 35, 3722 (1987).

[56] R. Rojas, F. Claro, and R. Fuchs, Phys. Rev. B 37, 6799 (1988).

[57] M. S. Yeung and T. K. Gustafson, Phys. Rev. A 54, 5227 (1996).

[58] V. V. Klimov and M. Ducloy, Phys. Rev. A 69, 013812 (2004).

[59] Y. N. Chen, G. Y. Chen, D. S. Chuu, and T. Brandes, Phys. Rev. A 79, 033815 (2009).

[60] D. Dzsotjan, A. S. Sørensen, and M. Fleischhauer, Phys. Rev. B 82, 075427 (2010)

[61] A. Trügler and U. Hohenester, Phys. Rev. B 77, 115403 (2008).

[62] A. Archambault, F. Marquier, J.-J. Greffet, and C. Arnold, Phys. Rev. B 82, 035411 (2010).

[63] A. Gonzalez-Tudela, F. J. Rodríguez, L. Quiroga, and C. Tejedor, Phys. Rev. B 82, 115334 (2010).

[64] E. S. Andrianov, A. A. Pukhov, A. V. Dorofeenko, A. P. Vinogradov, and A. A. Lisyansky, Phys. Rev. B 85, 035405 (2012).

[65] C. Van Vlack, P. T. Kristensen, and S. Hughes, Phys. Rev. B 85, 075303 (2012).

[66] M. S. Tame, C. Lee, J. Lee, D. Ballester, M. Paternostro, A. V. Zayats, and M. S. Kim, Phys. Rev. Lett. 101, 190504 (2008).

[67] H.-P. Breuer and F. Petruccione, The Theory of Open Quantum Systems (Oxford University Press, Oxford, 2002).

[68] J. Lindhard, Kgl. Danske Videnskab. Selskab. Mat.-Fys. Medd. 28, 8 (1954).

[69] N. D. Mermin, Phys. Rev. B 1, 2362 (1970).

[70] N. W. Ashcroft and N. D. Mermin, Solid State Physics, (Thomson Learning, Toronto, 1976).

[71] L. V. Keldysh, D. A. Kirzhnitz, and A. A. Maradudin, The Dielectric Function of Condensed Systems (North-Holland, Amsterdam, 1989).

[72] G. Knight, Introductory Quantum Optics (Cambridge University Press, Cambridge, 2005).

[73] V. B. Berestetskii, L. P. Pitaevskii, and E. M. Lifshitz, Quantum Electrodynamics (Butterworth-Heinemann, Oxford, 1982). 
[74] E. M. Lifshitz and L. P. Pitaevskii, Physical Kinetics (Pergamon, New York, 1981).

[75] M. Wilkinson and B. Mehlig, J. Phys.: Condens. Matter 12, 10481 (2000).

[76] R. H. Ritchie and A. L. Marusak, Surf. Sci. 4, 234 (1966).
[77] A. Thränhardt, C. Ell, G. Khitrova, and H. M. Gibbs, Phys. Rev. B 65, 035327 (2002).

[78] C. Cohen-Tannoudji, J. Dupont-Roc, and G. Grynberg, AtomPhoton Interactions (Wiley, New York, 1992).

[79] V. P. Drachev, U. K. Chettiar, A. V. Kildishev, H.-K. Yuan, W. Cai, and V. M. Shalaev, Opt. Express 16, 1186 (2008). 